\title{
Discovery and Synthesis of HIV Integrase Inhibitors: Development of Potent and Orally Bioavailable N-Methyl Pyrimidones
}

Cristina Gardelli, ${ }^{\S}$ Emanuela Nizi, ${ }^{\S}$ Ester Muraglia, ${ }^{\S}$ Benedetta Crescenzi, ${ }^{\S}$ Marco Ferrara, ${ }^{\S}$ Federica Orvieto, ${ }^{\S}$ Paola Pace, ${ }^{\S}$ Giovanna Pescatore, ${ }^{\S}$ Marco Poma, ${ }^{\S}$ Maria del Rosario Rico Ferreira, ${ }^{\S}$ Rita Scarpelli, ${ }^{\S}$ Carl F. Homnick, ${ }^{\theta}$ Norihiro Ikemoto, ${ }^{\varnothing}$ Anna Alfieri, ${ }^{\S}$ Maria Verdirame, ${ }^{\S}$ Fabio Bonelli, ${ }^{\S}$ Odalys Gonzalez Paz, ${ }^{\S}$ Marina Taliani, ${ }^{\S}$ Edith Monteagudo, ${ }^{\S}$ Silvia Pesci, ${ }^{\S}$ Ralph Laufer,${ }^{\S}$ Peter Felock, ${ }^{\ddagger}$ Kara A. Stillmock, Daria Hazuda, ${ }^{*}$ Michael Rowley, ${ }^{\S}$ Vincenzo Summa ${ }^{\S}$

${ }^{\S}$ Departments of Medicinal Chemistry and Pharmacology - Istituto Di Ricerche Di Biologia Molecolare, P. Angeletti S.p.A. (IRBM-MRL Rome), Via Pontina Km 30,600, 00040 Pomezia, Italy. ${ }^{\ominus}$ Department of Medicinal Chemistry MRL-West Point, PA, USA. ${ }^{\varnothing}$ Department of Process Research MRL-Rahway, NJ, USA. ${ }^{\ddagger}$ Department of Antiviral Research MRL-West Point, PA, USA.

RECEIVED DATE (to be automatically inserted after your manuscript is accepted if required according to the journal that you are submitting your paper to)

*To whom correspondence should be addressed. Phone: +39-0691093279; Fax: +39-0691093654. E-mail: cristina_gardelli@merck.com.

\section{Supporting Informations}

Scheme 1. ${ }^{a}$ Synthesis of compound 66d. 


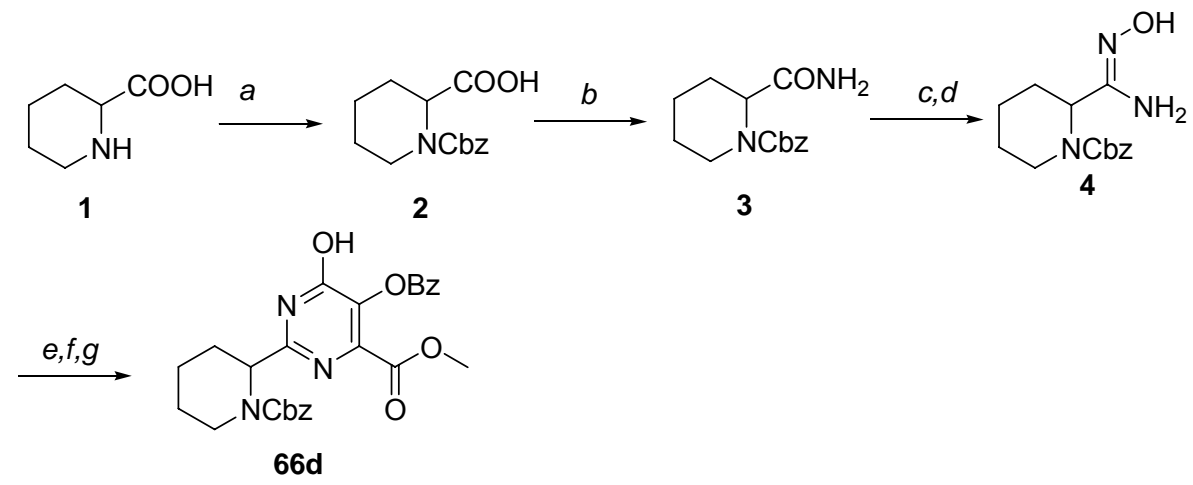

${ }^{a}$ Reagents and conditions: (a) $\mathrm{BnOCOCl}, \mathrm{Na}_{2} \mathrm{CO}_{3}, \mathrm{H}_{2} \mathrm{O}, 12 \mathrm{~h}$, r.t.; (b) $\mathrm{NH}_{3} 0.5 \mathrm{M}$ in dioxane, EDCI, HOBt, dioxane; (c) TFAA, $\mathrm{Et}_{3} \mathrm{~N}, \mathrm{DCM}$; (d) $\mathrm{NH}_{2} \mathrm{OH} \cdot \mathrm{HCl}, \mathrm{Et}_{3} \mathrm{~N}, \mathrm{EtOH}$; (e) DMAD, $\mathrm{CHCl}_{3}$, reflux, $0.5 \mathrm{~h}$; (f) $o$ xylenes, $150^{\circ} \mathrm{C} ;(\mathrm{g}) \mathrm{Bz} 2 \mathrm{O}$, Py.

1-[(Benzyloxy)carbonyl]piperidine-2-carboxylic acid (2). To a stirred solution of piperidine-2carboxylic acid and sodium carbonate (1.5 eq) in water cooled in an ice bath, benzylchloroformate (1.2 eq) was added dropwise with vigorous stirring. The mixture was stirred overnight at r.t.. The basic aqueous mixture was extracted two times with $\mathrm{Et}_{2} \mathrm{O}$. The separated alkaline layer was acidified with $1 \mathrm{~N} \mathrm{HCl}$ to $\mathrm{pH}$ 1 and extracted with AcOEt. The extract was washed with brine, dried $\left(\mathrm{Na}_{2} \mathrm{SO}_{4}\right)$, filtered and concentrated to 2, obtained as a white solid in $79 \%$ yield. ${ }^{1} \mathrm{H}$ NMR $\left(\mathrm{CDCl}_{3}, 400 \mathrm{MHz}\right) \delta$ 7.38-7.33 (m, 5H), 5.22-5.17 (m, 2H), 5.02-4.92 (m, 1H), $4.06(\mathrm{~d}, \mathrm{~J}=12.6 \mathrm{~Hz}, 1 \mathrm{H}), 3.06(\mathrm{dt}, \mathrm{J}=32 \mathrm{~Hz}, 12.6 \mathrm{~Hz}, 1 \mathrm{H}), 2.28(\mathrm{t}, \mathrm{J}=13.0$ $\mathrm{Hz}, 1 \mathrm{H}), 1.74-1.65$ (m, $3 \mathrm{H}), 1.47-1.30$ (m, $2 \mathrm{H})$.

Benzyl 2-(aminocarbonyl)piperidine-1-carboxylate (3). Compound 2 was charged in a flask and $\mathrm{NH}_{3}$ was added ( $2.5 \mathrm{eq})$ as a $0.5 \mathrm{M}$ solution in dioxane. To the stirred solution were added EDCI (1.3 eq.) and HOBt (1.3 eq.), and the reaction mixture was stirred overnight at r.t.. After evaporation of dioxane, the residue was poured into AcOEt and washed with $\mathrm{HCl} 1 \mathrm{~N}$, saturated $\mathrm{NaHCO}_{3}$ and brine. The organic extract was dried $\left(\mathrm{Na}_{2} \mathrm{SO}_{4}\right)$, filtered and concentrated to 3 obtained as a white solid in quantitative yield. ${ }^{1} \mathrm{H}$ NMR $\left(\mathrm{DMSO}_{d \sigma}, 400 \mathrm{MHz}\right) \delta 7.36$ (bs, 5H), 7.05 (bs, 2H), 5.06 (bs, 2H), 4.57 (d, J = 4.2 Hz, 1H), 3.89 $(\mathrm{d}, \mathrm{J}=13.1 \mathrm{~Hz}, 1 \mathrm{H}), 3.15-2.93(\mathrm{~m}, 1 \mathrm{H}), 2.07(\mathrm{~m}, 1 \mathrm{H}), 1.33-1.28(\mathrm{~m}, 3 \mathrm{H}), 1.32-1.92(\mathrm{~m}, 2 \mathrm{H})$.

Benzyl 2-[(Z)-amino(hydroxyimino)methyl]piperidine-1-carboxylate (4). To a stirred solution of compound 3 and $\mathrm{Et}_{3} \mathrm{~N}$ (3 eq) in DCM, trifluoroacetic anhydride (1.5 eq.) were added dropwise with an external cooling. Reaction mixture was stirred at r.t. for $1 \mathrm{~h}$. The solution was partitioned between DCM and $\mathrm{HCl} 1 \mathrm{~N}$, the organic phase was washed with brine, dried $\left(\mathrm{Na}_{2} \mathrm{SO}_{4}\right)$, filtered and concentrated to an oily residue containing the expected nitrile. Formation of the corresponding amidoxime was accomplished by refluxing this residue in absolute ethanol in the presence of $\mathrm{Et}_{3} \mathrm{~N}$ (3 eq.) and hydroxylamine 
hydrochloride (1.5 eq.) . After $3 \mathrm{~h}$ the reaction mixture was allowed to cool to r.t. and concentrated, and the residue triturated with water and dried to obtain compound 4. ${ }^{1} \mathrm{H}$ NMR $\left(\mathrm{DMSO}_{d \sigma}, 400 \mathrm{MHz}\right) \delta 9.17$ (s, $1 \mathrm{H}), 7.37-7.28$ (bs, 5H), 5.26 (bs, 2H), 5.10-5.00 (m, 2H), 4.72 (bs, 1H), 3.87 (d, J = 16.0 Hz, 1H), 3.10$2.98(\mathrm{~m}, 1 \mathrm{H}), 2.49-2.48(\mathrm{~m}, 1 \mathrm{H}), 1.58-1.28(\mathrm{~m}, 5 \mathrm{H})$.

Methyl 5-(benzoyloxy)-2-\{1-[(benzyloxy)carbonyl]piperidin-2-yl\}-6-hydroxypyrimidine-4carboxylate (66d). Compound 4 was suspended in chloroform and the minimal amount of methanol to allow complete solubilization was added. Dimethylacetylenedicarboxylate (1.2 eq.) were added to the stirred solution and reaction was refluxed for $0.5 \mathrm{~h}$. After cooling at r.t., volatiles were evaporated and the residue was refluxed in xylene for $24 \mathrm{~h}$. The solvent was then evaporated and the dark oily residue was taken up into pyridine and stirred overnight in the presence of benzoic anhydride (2 eq.). Reaction mixture was poured into $\mathrm{HCl} 1 \mathrm{~N}$, extracted in EtOAc, washed with $\mathrm{NaHCO}_{3}$ sat.sol. and brine, dried $\left(\mathrm{Na}_{2} \mathrm{SO}_{4}\right)$, filtered and concentrated. Residue was purified by column chromatography $\left(\mathrm{SiO}_{2}\right.$, petroleum ether:EtOAc=6:4). Collection of the appropriate fractions afforded the title product 66d (about 30\% yield). ${ }^{1} \mathrm{H}$ NMR $\left(\mathrm{CDCl}_{3}, 400 \mathrm{MHz}\right) \delta 10.8(\mathrm{bs}, 1 \mathrm{H}), 8.19(\mathrm{~d}, \mathrm{~J}=7.4 \mathrm{~Hz}, 2 \mathrm{H}), 7.66(\mathrm{t}, \mathrm{J}=7.4 \mathrm{~Hz}, 1 \mathrm{H}), 7.52(\mathrm{t}, \mathrm{J}=$ $7.8 \mathrm{~Hz}, 2 \mathrm{H}), 7.37-7.34(\mathrm{~m}, 5 \mathrm{H}), 5.31(\mathrm{bs}, 1 \mathrm{H}), 5.22(\mathrm{~d}, \mathrm{~J}=12.2 \mathrm{~Hz}, 1 \mathrm{H}), 5.17(\mathrm{~d}, \mathrm{~J}=12.2 \mathrm{~Hz}, 1 \mathrm{H}), 4.14-$ $4.11(\mathrm{~m}, 1 \mathrm{H}), 3.83(\mathrm{~s}, 3 \mathrm{H}), 2.89(\mathrm{t}, \mathrm{J}=11.6 \mathrm{~Hz}, 1 \mathrm{H}), 2.55-2.52(\mathrm{~m}, 1 \mathrm{H}), 1.78-1.52(\mathrm{~m}, 5 \mathrm{H}) . \mathrm{MS} \mathrm{m} / \mathrm{z} 492$ $(\mathrm{M}+\mathrm{H})^{+}$.

Scheme 2. ${ }^{a}$ Synthesis of compound 78.

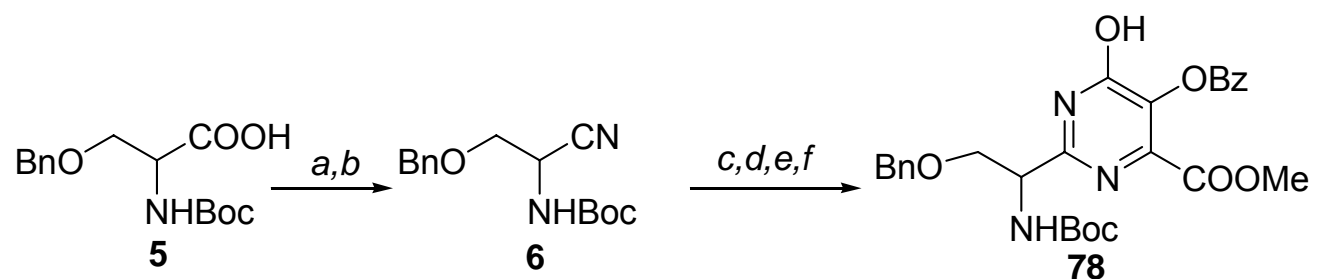

${ }^{a}$ Reagents and conditions: (a) $\mathrm{Boc}_{2} \mathrm{O}, \mathrm{NH}_{4} \mathrm{HCO}_{3}$, Py, r.t.; (b) TFAA, Et 3 N, DCM, r.t.; (c) $\mathrm{NH}_{2} \mathrm{OH} \cdot \mathrm{HCl}$, $\mathrm{KOH}, \mathrm{MeOH}$, r.t., $16 \mathrm{~h}$; (d) DMAD, $\mathrm{CHCl}_{3}, 60^{\circ} \mathrm{C}$, 3h; (e) $o$-xylenes, $130{ }^{\circ} \mathrm{C}, 12 \mathrm{~h}$; (f) $\mathrm{Bz} 2 \mathrm{O}, \mathrm{Py}, \mathrm{DCM}$. tert-Butyl [2-(benzyloxy)-1-cyanoethyl]carbamate (6). Carboxylic acid 5 (73 g, $247 \mathrm{mmol}$ ) was dissolved in pyridine $(400 \mathrm{ml})$, then $\mathrm{Boc}_{2} \mathrm{O}(64.7 \mathrm{~g}, 297 \mathrm{mmol})$ and $\mathrm{NH}_{4} \mathrm{HCO}_{3}(27.3 \mathrm{~g}, 346 \mathrm{mmol})$ were sequentially added, and the mixture was stirred at $\mathrm{rt}$ for $8 \mathrm{~h}$. After removing volatiles, the product was taken in EtOAc, the organic layer was washed with $1 \mathrm{~N} \mathrm{HCl}, \mathrm{NaHCO}_{3}$ sat. sol. and brine, dried $\left(\mathrm{Na}_{2} \mathrm{SO}_{4}\right)$, filtered and evaporated under vacuum. The crude was dissolved in DCM (400 ml) and $\mathrm{Et}_{3} \mathrm{~N}(104 \mathrm{~mL}, 744$ mmol) was added, the resulting mixture was cooled at $0{ }^{\circ} \mathrm{C}$, TFAA $(52.5 \mathrm{~mL}, 372 \mathrm{mmol})$ was added and 
the mixture was stirred at $\mathrm{rt}$ for $2 \mathrm{~h}$. Solvent was evaporated under vacuum, the residue was taken in EtOAc, the organic layer was washed with $1 \mathrm{~N} \mathrm{HCl}, \mathrm{NaHCO}_{3}$ sat. sol. and brine, dried $\left(\mathrm{Na}_{2} \mathrm{SO}_{4}\right)$, filtered and evaporated under vacuum to obtain crude compound 6 (78 g) that was used as such. ${ }^{1} \mathrm{H} \mathrm{NMR}\left(\mathrm{CDCl}_{3}\right.$, $300 \mathrm{MHz}) \delta 7.30-7.45$ (m, 5H), 5.21 (bs, 1H), 4.72 (bs, 1H), 4.63 (s, 2H), 3.73 (dd, J = 9.7, $3.5 \mathrm{~Hz}, 1 \mathrm{H})$, $3.64(\mathrm{dd}, \mathrm{J}=9.7,4.0 \mathrm{~Hz}, 1 \mathrm{H}), 1.46(\mathrm{~s}, 9 \mathrm{H}) . \mathrm{MS} m / z 277(\mathrm{M}+\mathrm{H})^{+}$.

Methyl 5-(benzoyloxy)-2-\{2-(benzyloxy)-1-[(tert-butoxycarbonyl)amino]ethyl\}-6hydroxypyrimidine-4-carboxylate (78). A solution of hydroxylamine hydrochloride (20.7 g, $297 \mathrm{mmol})$ in $\mathrm{MeOH}(180 \mathrm{~mL})$ was added at $0^{\circ} \mathrm{C}$ to a solution of $\mathrm{KOH}(16.7 \mathrm{~g}, 297 \mathrm{mmol})$ in $\mathrm{MeOH}(160 \mathrm{~mL})$. After stirring for $10 \mathrm{~min}, \mathrm{KCl}$ was filtered off and the filtrate was added to a solution of crude 6 (247 mmol) in $\mathrm{MeOH}(90 \mathrm{ml})$; the mixture was stirred at $\mathrm{rt}$ for $16 \mathrm{~h}$. Evaporation to dryness gave a residue that was treated $(247 \mathrm{mmol})$ as such with dimethyl acetylene dicarboxylate $(40.7 \mathrm{~g}, 286 \mathrm{mmol})$ in $\mathrm{CHCl}_{3}(600 \mathrm{ml})$ and stirred at $60^{\circ} \mathrm{C}$ for $3 \mathrm{~h}$. The crude product, obtained after evaporation of the solvent under vacuum, was used as such for the following cyclization reaction. The mixture of adducts (theor. 124 mmol, half of previous crude) in xylenes $(600 \mathrm{ml})$ was heated at reflux for $5 \mathrm{~h}$, then overnight at $130^{\circ} \mathrm{C}$. The mixture was evaporated under vacuum, the resulting crude was dissolved in dry DCM (400 ml), pyridine $(30 \mathrm{~mL}$, $375 \mathrm{mmol})$ and benzoic anhydride $(36.8 \mathrm{~g}, 163 \mathrm{mmol})$ were added. After stirring overnight at r.t. the mixture was evaporated under vacuum and the residue dissolved in EtOAc, washed with with $1 \mathrm{~N} \mathrm{HCl}$, $\mathrm{NaHCO}_{3}$ sat. sol. and brine, dried $\left(\mathrm{Na}_{2} \mathrm{SO}_{4}\right)$, filtered and evaporated under vacuum, to provide crude benzoate, which was purified by column chromatography $\left(\mathrm{SiO}_{2}\right.$, petroleum ether:EtOAc) to provide 13.9 g of 78 (21\% yield from 5, 6 steps). ${ }^{1} \mathrm{H}$ NMR $\left(\mathrm{CDCl}_{3}, 300 \mathrm{MHz}\right) \delta 10.55(\mathrm{bs}, 1 \mathrm{H}), 8.19(\mathrm{~d}, \mathrm{~J}=7.1 \mathrm{~Hz}$, 2H), $7.65(\mathrm{t}, \mathrm{J}=7.4 \mathrm{~Hz}, 1 \mathrm{H}), 7.50(\mathrm{t}, \mathrm{J}=7.6,2 \mathrm{H}), 7.25-7.38(\mathrm{~m}, 5 \mathrm{H}), 5.52(\mathrm{bd}, \mathrm{J}=4.6 \mathrm{~Hz}, 1 \mathrm{H}), 4.80(\mathrm{bs}$, $1 \mathrm{H}), 4.56(\mathrm{~s}, 2 \mathrm{H}), 3.96-4.15(\mathrm{~m}, 1 \mathrm{H}), 3.82(\mathrm{~s}, 3 \mathrm{H}), 3.76(\mathrm{dd}, \mathrm{J}=9.5,5.5 \mathrm{~Hz}, 1 \mathrm{H}), 1.45(\mathrm{~s}, 9 \mathrm{H}) . \mathrm{MS} m / z 524$ $(\mathrm{M}+\mathrm{H})^{+}$.

Scheme 3. ${ }^{a}$ Synthesis of compounds $\mathbf{8 9 g}$ and $\mathbf{8 9 h}$. 

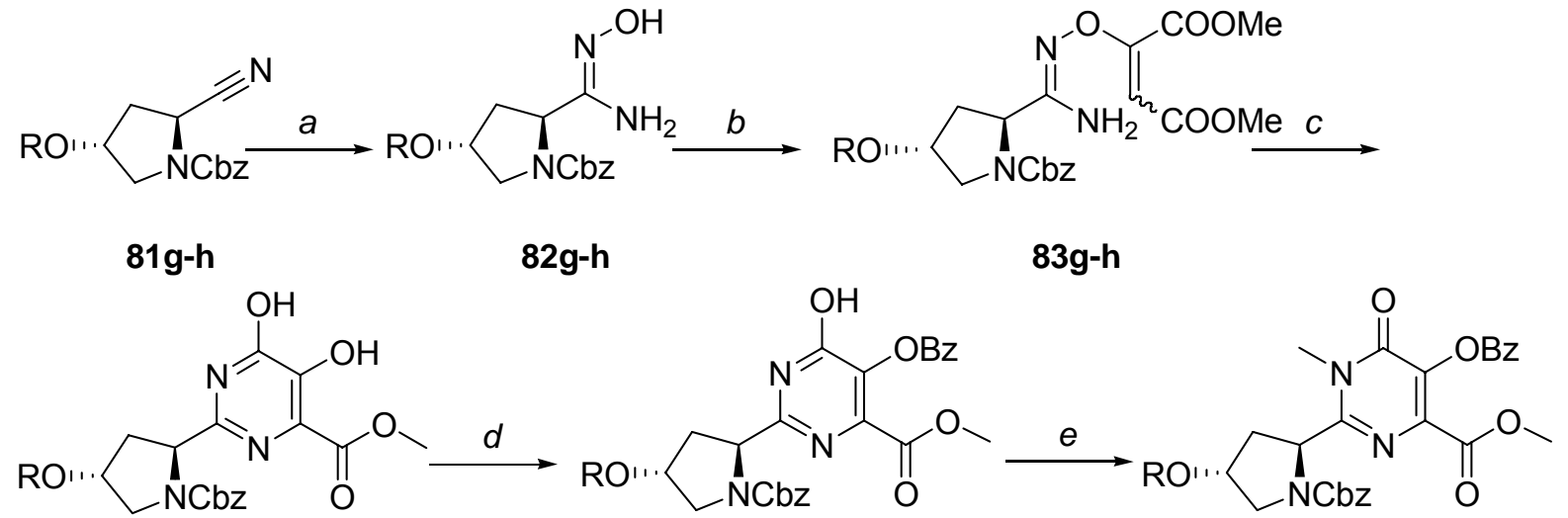

84g-h

85g-h

86g-h

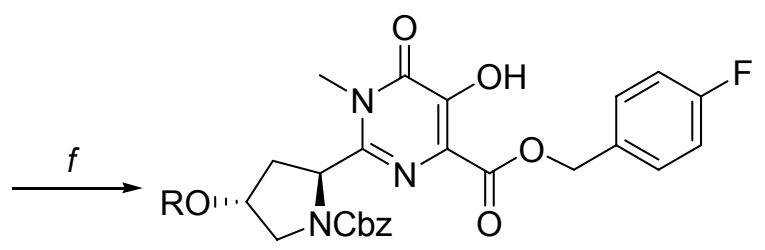

$$
\begin{array}{ll}
\text { 89g-h } & \text { h: } R=M e \\
\text { h: } R=E t
\end{array}
$$

${ }^{a}$ Reagents and conditions: (a) $\mathrm{NH}_{2} \mathrm{OH} \cdot \mathrm{HCl}, \mathrm{Et}_{3} \mathrm{~N}$, EtOH, $40{ }^{\circ} \mathrm{C}$, 4h; (b) DMAD, $\mathrm{CHCl}_{3}$, reflux, $1 \mathrm{~h}$; (c) $o$ xylenes, $150{ }^{\circ} \mathrm{C}$, $12 \mathrm{~h}$; (d) $\mathrm{Bz}_{2} \mathrm{O}$, Py, 3h; (e) $\mathrm{LiH}$, dioxane, $\mathrm{Me}_{2} \mathrm{SO}_{4}$; (f) 4F- $\mathrm{BnNH}_{2}, \mathrm{MeOH}$, reflux.

Benzyl-(2S,4R)-2-[amino(hydroxyimino)methyl]-4methoxypyrrolidine -1-carboxylate (82g). To the nitrile 81g (4.62 g, $17.8 \mathrm{mmol})$, dissolved in ethanol $(40 \mathrm{ml}), \mathrm{NH}_{2} \mathrm{OH} \cdot \mathrm{HCl}\left(1.6 \mathrm{~g}, 1.3\right.$ eq.) and $\mathrm{Et}_{3} \mathrm{~N}(3.66$ $\mathrm{ml}, 1.5$ eq.) were added. The mixture was stirred at $40^{\circ} \mathrm{C}$ for $4 \mathrm{~h}$ then at r.t. overnight. The mixture was concentrated in vacuo and the residue dissolved in ethyl acetate washed with brine, dried $\left(\mathrm{Na}_{2} \mathrm{SO}_{4}\right)$, filtered and concentrated to give the title product $\mathbf{8 2 g},(4.80 \mathrm{~g}, 92 \%) .{ }^{1} \mathrm{H}$ NMR $\left(\mathrm{DMSO}_{d 6}, 400 \mathrm{MHz}, 300\right.$ K) $\delta 9.05(\mathrm{bs}, 1 \mathrm{H}), 7.45-7.25(\mathrm{~m}, 5 \mathrm{H}), 5.4(\mathrm{bs}, 2 \mathrm{H}), 5.10(\mathrm{~d}, \mathrm{~J}=13 \mathrm{~Hz}, 1 \mathrm{H}), 5.03(\mathrm{~d}, \mathrm{~J}=13 \mathrm{~Hz}, 1 \mathrm{H}), 4.26$ (t, J = 7.4 Hz, 1H), 3.97 (bs, 1H), 3.63-3.45 (m, 2H), 3.22 (s, 3H) 2.3-2.03 (m, 2H).

\section{Dimethyl-2-\{[(amino-\{(2S,4R)-1-[(benzyloxy)carbonyl]-4-methoxypyrrolidin-2-}

yl\}methylidene)amino]oxy\}but-2-enedioate (83g). To compound 82g (4.80 g, $16.4 \mathrm{mmol})$, dissolved in chloroform (90 ml), dimethyl acetylendicarboxylate $(2.22 \mathrm{ml}, 1.1$ eq.) was added. The mixture was refluxed for $1 \mathrm{~h}$ and left stirring at $40{ }^{\circ} \mathrm{C}$ overnight. The chloroform was removed in vacuo and the crude was purified by column chromatography $\left(\mathrm{SiO}_{2}\right.$, petroleum ether:ethyl acetate=3:2) yielding compound 83g as a 7:3 mixture of isomers (4.26 g, $60 \%$ yield). ${ }^{1} \mathrm{H}$ NMR $\left(\mathrm{DMSO}_{d 6}, 400 \mathrm{MHz}\right) \delta 7.40-7.23(\mathrm{~m}, 5 \mathrm{H})$, 6.7-6.55 (2bs, $1.4 \mathrm{H}), 6.35-6.2(\mathrm{bs}, 0.6 \mathrm{H}), 5.61(\mathrm{~s}, 0.7 \mathrm{H}), 5.59(\mathrm{~s}, 0.3 \mathrm{H}), 5.08(\mathrm{~s}, 0.6 \mathrm{H}), 5.10(\mathrm{~d}, \mathrm{~J}=13 \mathrm{~Hz}$, 
0.7H), $5.02(\mathrm{~d}, \mathrm{~J}=13 \mathrm{~Hz}, 0.7 \mathrm{H}), 4.30-4.20(\mathrm{~m}, 1 \mathrm{H}), 3.97(\mathrm{bs}, 1 \mathrm{H}), 3.78$ (s, 2.1H), 3.73 (s, 0.9H), $3.62(\mathrm{~s}$, $0.9 \mathrm{H}), 3.59(\mathrm{~s}, 2.1 \mathrm{H}), 3.65-3.50(\mathrm{~m}, 2 \mathrm{H}), 3.22(\mathrm{~s}, 3 \mathrm{H}), 2.37-2.23(\mathrm{~m}, 1 \mathrm{H}), 2.10-1.95(\mathrm{~m}, 1 \mathrm{H})$.

Methyl 5-(benzoyloxy)-2-\{(2S,4R)-1-[(benzyloxy)carbonyl]-4-methoxypyrrolidin-2-yl\}-6hydroxypyrimidine-4-carboxylate (85g). Compound $\mathbf{8 3 g}$ (5.55 g, $12.8 \mathrm{mmol}$ ) was dissolved in xylene $(212 \mathrm{ml})$ and the solution stirred at $150{ }^{\circ} \mathrm{C}$ for $3 \mathrm{~h}$ and at r.t. overnight. Xylene was concentrated in vacuo. To the crude compound $\mathbf{8 4 g}$, dissolved in pyridine $(50 \mathrm{ml})$, benzoic anhydride $(3.75,1.3 \mathrm{eq}$.) was added, the reaction mixture was stirred at r.t. overnight. The solution was concentrated in vacuo and the crude dissolved in ethyl acetate washed with $\mathrm{HCl} 1 \mathrm{~N}, \mathrm{NaHCO}_{3}$ sat. sol. and brine, dried $\left(\mathrm{Na}_{2} \mathrm{SO}_{4}\right)$, filtered and evaporated in vacuo. The title product was obtained after purification by column chromatography $\left(\mathrm{SiO}_{2}\right.$, petroleum ether:ethyl acetate=9:1) yielding compound $\mathbf{8 5 g}(54 \%)$ as a 1:1 mixture of rotamers by NMR. ${ }^{1} \mathrm{H}$ NMR $\left(\mathrm{DMSO}_{d 6}, 400 \mathrm{MHz}\right) \delta 13.5(\mathrm{~s}, 1 \mathrm{H}), 8.09$ (t, J = 7.0 Hz, 2H), 7.82-7.75 (m, 1H), 7.66-7.61 (m, 2H), 7.40-7.25 (m, $4 \mathrm{H}), 7.12-7.06(\mathrm{~m}, 1 \mathrm{H}), 5.10(\mathrm{~s}, 1 \mathrm{H}), 5.09$ (d, J = 12.5 Hz, 0.5H), 4.88 (d, J = 12.5 Hz, $0.5 \mathrm{H}), 4.66(\mathrm{dd}, \mathrm{J}=16.2,8.0 \mathrm{~Hz}, 1 \mathrm{H}), 4.10-4.00(\mathrm{~m}, 1 \mathrm{H}), 3.74(\mathrm{~s}, 3 \mathrm{H}), 3.75-3.60(\mathrm{~m}, 2 \mathrm{H}), 3.25(\mathrm{~s}, 3 \mathrm{H})$, 2.45-2.40 (partially under DMSO) (m, 1H), 2.13-2.03 (m, 1H).

\section{Methyl-5-(benzoyloxy)-2-\{(2S,4R)-1-[(benzyloxy)carbonyl]-4-methoxypyrrolidin-2-yl\}-1-methyl-6-}

oxo-1,6-dihydropyrimidine-4-carboxylate (86g). To compound $\mathbf{8 5 g}(3.47 \mathrm{~g}, 6.84 \mathrm{mmol})$, dissolved in dioxane $(31 \mathrm{ml})$, $\mathrm{LiH}\left(0.12 \mathrm{~g}, 2.2\right.$ eq.) was added and the reaction mixture stirred at $38^{\circ} \mathrm{C}$ for 40 minutes. The temperature was raised to $60^{\circ} \mathrm{C}$ and dimethyl sulphate $(1.96 \mathrm{ml}, 3$ eq. $)$ was added dropwise. After two $\mathrm{h}$ the reaction mixture was cooled down to $0^{\circ} \mathrm{C}$ and $\mathrm{HCl} 1 \mathrm{~N}$ was added to quench the reaction. The reaction mixture was extracted with ethyl acetate and the organic phase washed with $\mathrm{HCl} 1 \mathrm{~N}$, saturated aqueous $\mathrm{NaHCO}_{3}$ and brine, dried $\left(\mathrm{Na}_{2} \mathrm{SO}_{4}\right)$, filtered and concentrated in vacuo. The title product was isolated by column chromatography $\left(\mathrm{SiO}_{2}\right.$, petroleum ether:ethyl acetate=7:3) yielding compound 86g as a 1:1 mixture of rotamers by NMR, (4.62 g, 63\% yield). ${ }^{1} \mathrm{H}$ NMR $\left(\mathrm{DMSO}_{d 6}, 400 \mathrm{MHz}\right) \delta 8.08(\mathrm{t}, \mathrm{J}=6.8$ $\mathrm{Hz}, 2 \mathrm{H}), 7.82-7.75(\mathrm{~m}, 1 \mathrm{H}), 7.66-7.61(\mathrm{~m}, 2 \mathrm{H}), 7.38-7.22(\mathrm{~m}, 4 \mathrm{H}), 7.08-7.02(\mathrm{~m}, 1 \mathrm{H})$, 5.18-5.12 (m, 1H), $5.13(\mathrm{~d}, \mathrm{~J}=13.1 \mathrm{~Hz}, 0.5 \mathrm{H}), 5.07(\mathrm{~d}, \mathrm{~J}=13.1 \mathrm{~Hz}, 0.5 \mathrm{H}), 5.07(\mathrm{~d}, \mathrm{~J}=12.4 \mathrm{~Hz}, 0.5 \mathrm{H}), 4.84(\mathrm{~d}, \mathrm{~J}=12.4 \mathrm{~Hz}$, $0.5 \mathrm{H}), 4.08-4.17(\mathrm{~m}, 1 \mathrm{H}), 3.73(\mathrm{~s}, 3 \mathrm{H}), 3.75-3.55(\mathrm{~m}, 2 \mathrm{H}), 3.65(\mathrm{~s}, 1.5 \mathrm{H}), 3.44(\mathrm{~s}, 1.5 \mathrm{H}), 3.26(\mathrm{~s}, 3 \mathrm{H})$, 2.62-2.52 (partially under DMSO) (m, 1H), 2.30-2.15 (m, 1H). MS m/z $522(\mathrm{M}+\mathrm{H})^{+}$.

\section{Benzyl-(2S,4R)-2-(4-\{[(4-fluorobenzyl)amino]carbonyl\}-5-hydroxy-1-methyl-6-oxo-1,6-}

dihydropyrimidin-2-yl)-4-methoxypyrrolidine-1-carboxylate (89g). To compound 86g (0.5 g, 0.96 mmol) dissolved in methanol (30 ml), 4-F-benzylamine $(0.36 \mathrm{~g}, 3$ eq.) was added. The reaction mixture was stirred at reflux overnight. Methanol was removed in vacuo and the residue triturated with ethyl ether 
to give the title product $\mathbf{8 9 g}$ as a 4:6 mixture of rotamers by NMR: ${ }^{1} \mathrm{H}$ NMR $\left(\mathrm{DMSO}_{d}+\mathrm{TFA}, 400 \mathrm{MHz}\right) \delta$ $14.0(\mathrm{bs}, 1 \mathrm{H}), 8.92(\mathrm{t}, \mathrm{J}=6.4 \mathrm{~Hz}, 0.4 \mathrm{H}), 8.73(\mathrm{t}, \mathrm{J}=5.9 \mathrm{~Hz}, 0.6 \mathrm{H}), 7.35-7.25(\mathrm{~m}, 4 \mathrm{H}), 7.20-7.05(\mathrm{~m}, 4 \mathrm{H})$, $6.93(\mathrm{~d}, \mathrm{~J}=7.5 \mathrm{~Hz}, 1 \mathrm{H}), 5.09-4.95(\mathrm{~m}, 1 \mathrm{H}), 5.09$ (d, J = 12.3 Hz, 0.6H), 4.75 (d, J = 12.3 Hz, 0.6H), 5.05 $(\mathrm{d}, \mathrm{J}=13 \mathrm{~Hz}, 0.4 \mathrm{H}), 4.98$ (d, J = $13 \mathrm{~Hz}, 0.4 \mathrm{H}), 4.52-4.43(\mathrm{~m}, 2 \mathrm{H}), 4.12-4.06$ (bm, 0.4H), 4.06-4.02 (bm, 0.6H), $3.87(\mathrm{dd}, \mathrm{J}=11.5,4.5 \mathrm{~Hz}, 0.4 \mathrm{H}), 3.84(\mathrm{dd}, \mathrm{J}=12.0,2.7 \mathrm{~Hz}, 0.6 \mathrm{H}), 3.65-3.55(\mathrm{~m}, 1 \mathrm{H}), 3.59(\mathrm{~s}$, $1.2 \mathrm{H}), 3.41(\mathrm{~s}, 1.8 \mathrm{H}), 3.25(\mathrm{~s}, 3 \mathrm{H}), 2.45-2.40$ (partially under DMSO) (m, 1H), 2.30-2.15 (m, 1H). MS m/z $511(\mathrm{M}+\mathrm{H})^{+}$.

Benzyl (2S,4R)-4-ethoxy-2-(4-\{[(4-fluorobenzyl)amino]carbonyl\}-5-hydroxy-1-methyl-6-oxo-1,6dihydropyrimidin-2-yl)pyrrolidine-1-carboxylate (89h). Following the same steps described for compound $\mathbf{8 9 g}$, compound $\mathbf{8 9 h}$ was obtained by $\mathbf{8 5 h}$ as a $2: 3$ mixture of rotamers by NMR. ${ }^{1} \mathrm{H}$ NMR $\left(\mathrm{DMSO}_{d 6}, 400 \mathrm{MHz}\right) \delta 12.15(\mathrm{~s}, 0.4 \mathrm{H}), 12.09(\mathrm{~s}, 0.6 \mathrm{H}), 8.89(\mathrm{t}, \mathrm{J}=6.4 \mathrm{~Hz}, 0.4 \mathrm{H}), 8.69(\mathrm{t}, \mathrm{J}=5.9 \mathrm{~Hz}$, 0.6H), 7.49-7.23 (m, 4H), 7.19-7.05 (m, 4H), $6.91(\mathrm{~d}, \mathrm{~J}=7.5 \mathrm{~Hz}, 1 \mathrm{H}), 5.11-4.95(\mathrm{~m}, 2.4 \mathrm{H}), 4.75(\mathrm{~d}, \mathrm{~J}=$ $12.3 \mathrm{~Hz}, 0.6 \mathrm{H}), 4.55-4.45(\mathrm{~m}, 2 \mathrm{H}), 4.19(\mathrm{~m}, 0.4 \mathrm{H}), 4.12(\mathrm{~m}, 0.6 \mathrm{H}), 3.93-3.81(\mathrm{~m}, 1 \mathrm{H}), 3.60-3.41(\mathrm{~m}, 6 \mathrm{H})$, 2.45-2.12 (partially under DMSO) (m, 2H), 1.12 (t, J=7.2 Hz, 3H). MS m/z $525(\mathrm{M}+\mathrm{H})^{+}$.

Scheme 4. ${ }^{a}$ Synthesis of compound $85 i$.

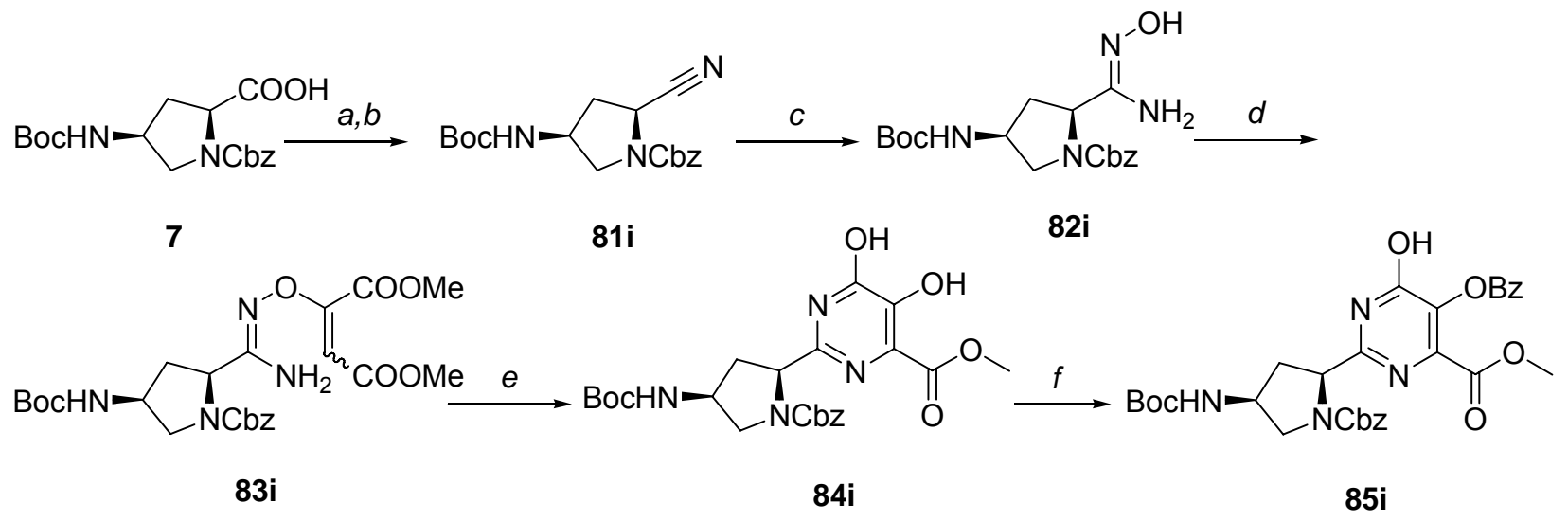

${ }^{a}$ Reagents and conditions: (a) $\mathrm{Boc}_{2} \mathrm{O}, \mathrm{NH}_{4} \mathrm{HCO}_{3}$, dioxane, (b) TFAA, Et 3 N, DCM; (c) $\mathrm{NH}_{2} \mathrm{OH} \cdot \mathrm{HCl}, \mathrm{Et}_{3} \mathrm{~N}$, EtOH; (d) DMAD, $\mathrm{CHCl}_{3}$, reflux, 2h; (e) o-xylenes, reflux, 3.5h; (f) $\mathrm{Bz} 2 \mathrm{O}, \mathrm{Py}$.

Benzyl (2S,4S)-4-[(tert-butoxycarbonyl)amino]-2-cyanopyrrolidine-1-carboxylate (81i). A stirred solution of compound $7^{1},(6.7 \mathrm{~g}, 18.4 \mathrm{mmol})$ in dioxane $(30 \mathrm{~mL})$ was treated with pyridine $(1 \mathrm{~mL}, 0.7 \mathrm{eq}$. and $\mathrm{Boc}_{2} \mathrm{O}$ (5.2 g, 1.3 eq.), then ammonium bicarbonate (1.8 g, 1.26 eq.) was added and the mixture was stirred at r.t. overnight. Dioxane was concentrated and the residue was taken up in ethyl acetate, washed with water and brine and dried $\left(\mathrm{Na}_{2} \mathrm{SO}_{4}\right)$ to give, after filtration and concentration the primary amide that was submitted to the next step without further purification, $6.8 \mathrm{~g}$ ( $>98 \%$ yield $).{ }^{1} \mathrm{H}$ NMR $\left(\mathrm{DMSO}_{d 6}, 300\right.$ 
$\mathrm{MHz}, 340 \mathrm{~K}) \delta 7.36$ (m, $5 \mathrm{H}), 7.10-6.63$ (m, 2H), 5.07 (s, 2H), 4.19 (br m, 1H), 4.09-3.96 (m, 1H), 3.72 (dd, J = 10.6, 6.7 Hz, 1H), 3.20 (dd, J = 10.6, $6.0 \mathrm{~Hz}, 1 \mathrm{H}), 2.44$ (m, 1H), $1.76(\mathrm{~m}, 1 \mathrm{H}), 1.40$ (s, 9H). MS $m / z 364(\mathrm{M}+\mathrm{H})^{+}$. The primary amide $(6.8 \mathrm{~g}, 18.4 \mathrm{mmol})$ in $\mathrm{DCM}(35 \mathrm{~mL})$ was treated at $0{ }^{\circ} \mathrm{C}$ with $\mathrm{Et}_{3} \mathrm{~N}$ (5.4 mL, 2.1 eq.) and trifluoroacetic anhydride $(2.9 \mathrm{~mL}, 1.1$ eq.) was added dropwise. The reaction mixture was stirred at r.t. for 1 h. Volatiles were removed i.v., the residue was dissolved in EtOAc, washed with $\mathrm{HCl} 1 \mathrm{~N}$ and brine, dried $\left(\mathrm{Na}_{2} \mathrm{SO}_{4}\right)$ and filtered to give, after concentration, a residue which was purified by column chromatography: $\left(\mathrm{SiO}_{2}\right.$, petroleum ether:ethyl acetate $\left.=4: 1\right)$ yielding compound 81i, (4.8 g, 76\% yield). ${ }^{1} \mathrm{H}$ NMR $\left(\mathrm{DMSO}_{d 6}, 300 \mathrm{MHz}, 340 \mathrm{~K}\right) \delta 7.41$ (m, $\left.5 \mathrm{H}\right), 7.03$ (m, 1H), 5.16 (s, 2H), 4.73 (br m, 1H), 4.07-3.99 (m, 1H), 3.63 (dd, J = 10.7, $6.3 \mathrm{~Hz}, 1 \mathrm{H}), 3.35$ (dd, J = 10.7, $4.9 \mathrm{~Hz}, 1 \mathrm{H}), 2.47$ (m, 1H), 2.30-2.24 (m, 1H), $1.41(\mathrm{~s}, 9 \mathrm{H}) . \mathrm{MS} \mathrm{m} / z 346(\mathrm{M}+\mathrm{H})^{+}$.

\section{Benzyl (2S,4S)-2-[(Z)-amino(hydroxyimino)methyl]-4-[(tert-butoxycarbonyl)amino]pyrrolidine-1-} carboxylate (82i). The nitrile 81i $(4.8 \mathrm{~g}, 13.9 \mathrm{mmol})$ dissolved in absolute ethanol $(40 \mathrm{~mL})$ was treated with triethyl amine (3.3 mL, 1.7 eq.) and hydroxylamine hydrochloride (1.4 g, 1.4 eq.). The reaction mixture was stirred at $50^{\circ} \mathrm{C}$ for $3 \mathrm{~h}$ and then the solvent was removed under reduced pressure. The residue was taken up in water and EtOAc and the aqueous layer was extracted with EtOAc two times. The organic phase were washed with brine, dried $\left(\mathrm{Na}_{2} \mathrm{SO}_{4}\right)$ to give, after filtration and concentration, compound 82i, that was submitted to the next step without further purification, $5.1 \mathrm{~g}$ (97\% yield). ${ }^{1} \mathrm{H} \mathrm{NMR}\left(\mathrm{DMSO}_{d 6}\right.$, $300 \mathrm{MHz}, 340 \mathrm{~K}) \delta 8.96(\mathrm{~s}, 1 \mathrm{H}), 7.36(\mathrm{~m}, 5 \mathrm{H}), 6.81(\mathrm{~m}, 1 \mathrm{H}), 5.31(\mathrm{bs}, 2 \mathrm{H}), 5.07(\mathrm{~m}, 2 \mathrm{H}), 4.31(\mathrm{t}, \mathrm{J}=7.1$ $\mathrm{Hz}, 1 \mathrm{H}), 4.03-3.92(\mathrm{~m}, 1 \mathrm{H}), 3.80(\mathrm{dd}, \mathrm{J}=10.6,6.9 \mathrm{~Hz}, 1 \mathrm{H}), 3.20-3.14$ (obscured by residual water, $1 \mathrm{H})$, $2.36(\mathrm{~m}, 1 \mathrm{H}), 1.94(\mathrm{~m}, 1 \mathrm{H}), 1.40(\mathrm{~s}, 9 \mathrm{H}) . \mathrm{MS} \mathrm{m} / z 379(\mathrm{M}+\mathrm{H})^{+}$.

\section{Methyl}

5-(benzoyloxy)-2-\{(2S,4S)-1-[(benzyloxy)carbonyl]-4-[(tertbutoxycarbonyl)amino]pyrrolidin-2-yl\}-6-hydroxy-pyrimidine-4-carboxylate (85i). A solution of dimethylacetylene dicarboxylate $(2.0 \mathrm{ml}, 1.2$ eq.) and compound $\mathbf{8 2 i}(5.1 \mathrm{~g}, 13.5 \mathrm{mmol})$ in chloroform (20 $\mathrm{ml}$ ) was refluxed for $2 \mathrm{~h}$. The chloroform was evaporated under vacuum and the residue was purified by column chromatography $\left(\mathrm{SiO}_{2}\right.$, petroleum ether:ethyl acetate=from 4:3 to 1:1) yielding the desired adducts 83i as a mixture of configurational isomers $\mathrm{E}$ and $\mathrm{Z}, 4.0 \mathrm{~g}(58 \%$ yield $)$. MS $\mathrm{m} / \mathrm{z} 521(\mathrm{M}+\mathrm{H})^{+}$. The mixture of adducts $83 \mathbf{i}(4.0 \mathrm{~g}, 7.7 \mathrm{mmol})$ was refluxed in $o$-xylene $(45 \mathrm{ml})$ for $3.5 \mathrm{~h}$. Volatiles were evaporated i.v. to give the desired compound 84i that was submitted to the next step without purification. The dihydroxypyrimidine 84i in pyridine $(40 \mathrm{~mL})$ was treated with benzoic anhydride $(2.3 \mathrm{~g}, 10 \mathrm{mmol})$ and the reaction mixture was stirred at r.t. overnight. Pyridine was concentrated and the residue was taken up in ethyl acetate and washed with $\mathrm{HCl} 1 \mathrm{~N}$ and brine. The organic phase was dried $\left(\mathrm{Na}_{2} \mathrm{SO}_{4}\right)$ and filtered to 
give after concentration a crude that was purified by column chromatography $\left(\mathrm{SiO}_{2}\right.$, petroleum ether:ethyl acetate $=$ from $1: 1$ to $1: 4)$ yielding compound $85 \mathrm{i}\left(2.0 \mathrm{~g}, 42 \%\right.$ yield). ${ }^{1} \mathrm{H}$ NMR $\left(\mathrm{DMSO}_{d 6}, 400 \mathrm{MHz}, 340 \mathrm{~K}\right)$ $\delta 13.24$ (bs, 1H), 8.07 (d, J = 7.2 Hz, 2H), 7.76 (t, J = 7.5 Hz, 1H), 7.62 (t, J = $7.7 \mathrm{~Hz}, 2 \mathrm{H}$ ), 7.31 (bm, 5 H), $6.93(\mathrm{bm}, 1 \mathrm{H}), 5.08(\mathrm{bm}, 2 \mathrm{H}), 4.73(\mathrm{t}, \mathrm{J}=7.4 \mathrm{~Hz}, 1 \mathrm{H}), 4.05(\mathrm{dd}, \mathrm{J}=14.2,6.6 \mathrm{~Hz}, 1 \mathrm{H}), 3.85-3.80(\mathrm{~m}$, $1 \mathrm{H}), 3.75(\mathrm{~s}, 3 \mathrm{H}), 3.39$ (dd, J = 10.5, $6.6 \mathrm{~Hz}, 1 \mathrm{H}), 2.57(\mathrm{~m}, 1 \mathrm{H}), 2.01-1.95(\mathrm{~m}, 1 \mathrm{H}), 1.39(\mathrm{~s}, 9 \mathrm{H}) . \mathrm{MS} \mathrm{m} / \mathrm{z}$ $593(\mathrm{M}+\mathrm{H})^{+}$.

Scheme 5. ${ }^{a}$ Synthesis of compound 90 .<smiles>COC(=O)[C@H]1C[C@@H](O)CN1C(=O)[O-]</smiles>

97

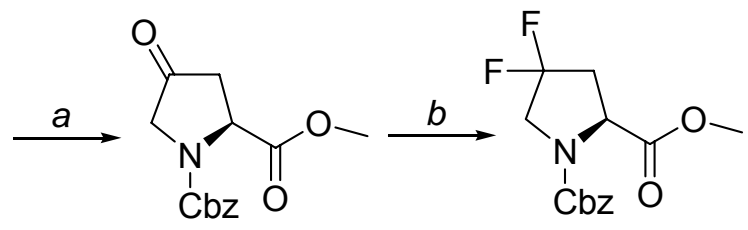

99

8<smiles>CC(C)[C@H]1N(C(=O)[O-])[C@H](C(=O)O)CC1(F)F</smiles>

9

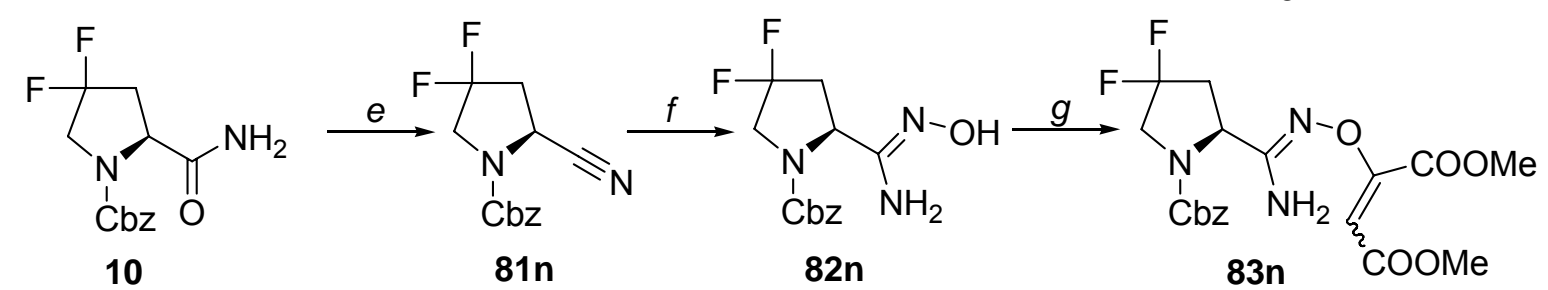<smiles>CCCCOc1c(O)nc(C2CC(F)(F)CN2C(=O)c2ccccc2)nc1C(=O)O</smiles>

$84 n$

$85 n$

$86 n$<smiles>CC(C)C(=O)N1CC(F)(F)C[C@H]1c1nc(C(=O)NCc2ccc(F)cc2)c(O)c(=O)n1C</smiles>

$89 n$<smiles>Cn1c([C@@H]2CC(F)(F)CN2)nc(C(=O)NCc2ccc(F)cc2)c(O)c1=O</smiles>

90n

${ }^{a}$ Reagents and conditions: (a) DMSO, $(\mathrm{COCl})_{2}, \mathrm{Et}_{3} \mathrm{~N}, \mathrm{DCM},-78^{\circ} \mathrm{C}$; (b) DAST, DCM, $-78{ }^{\circ} \mathrm{C}$; (c) $\mathrm{NaOH}$, $\mathrm{MeOH}$; (d) $\mathrm{NH}_{4} \mathrm{HCO}_{3}, \mathrm{Boc}_{2} \mathrm{O}$, Py, dioxane; (e) TFAA, $\mathrm{Et}_{3} \mathrm{~N}, \mathrm{DCM}$; (f) $\mathrm{NH}_{2} \mathrm{OH} \cdot \mathrm{HCl}, \mathrm{Et}_{3} \mathrm{~N}, \mathrm{EtOH}$, reflux, 5h; (g) DMAD, $\mathrm{CHCl}_{3}$, reflux, $1 \mathrm{~h}$; (h) $o$-xylenes, $158^{\circ} \mathrm{C}$; (i) $\mathrm{Bz} 2 \mathrm{O}$, Py, 3h; (l) $\mathrm{LiH}$, dioxane, $\mathrm{Me}_{2} \mathrm{SO}_{4}$; (m) 4-F-BnNH${ }_{2}, \mathrm{MeOH}$; (n) $\mathrm{H}_{2}, \mathrm{Pd} / \mathrm{C}, \mathrm{MeOH}$. 
(2S)-1-Benzyl-2-methyl-4-oxopyrrolidine-1,2-dicarboxylate (99). A solution of dimethyl sulfoxide $(2.05 \mathrm{ml}, 26.54 \mathrm{mmol})$ in dry DCM $(8.1 \mathrm{ml})$ was added dropwise to a stirred solution of oxalyl chloride $(1.14 \mathrm{ml}, 13.3 \mathrm{mmol})$ in dry DCM $(10.6 \mathrm{ml})$ at $-78{ }^{\circ} \mathrm{C}$ under $\mathrm{N}_{2}$ atmosphere. After $15 \mathrm{~min}$, a solution of the commercially available (2S,4R)-1-benzyl-2-methyl-4-hydroxypyrrolidine-1,2-dicarboxylate 97 (3.37 g, $12.1 \mathrm{mmol})$ in dry DCM $(16 \mathrm{ml})$ was added slowly, and stirring was continued for $30 \mathrm{~min}$ at $-78{ }^{\circ} \mathrm{C}$. After addition of $\mathrm{Et}_{3} \mathrm{~N}(8.4 \mathrm{ml}, 60.35 \mathrm{mmol})$, the mixture was gradually warmed up to r.t.. The mixture was quenched with water and aqueous layer was separated and extracted with DCM. The extract was washed with brine and dried $\left(\mathrm{Na}_{2} \mathrm{SO}_{4}\right)$. Concentration of the solvent in vacuo gave the title product 99 as a yellow oil (90\% yield) which was used in the next step without any further purification. ${ }^{1} \mathrm{H}$ NMR $\left(\mathrm{DMSO}_{d \sigma}+\mathrm{TFA}, 400 \mathrm{MHz}, 330 \mathrm{~K}\right) \delta$ 7.40-7.32 (m, 5H), 5.20-5.09 (m, 2H), $4.79(\mathrm{~d}, \mathrm{~J}=9.7 \mathrm{~Hz}, 1 \mathrm{H}), 3.95$ $(\mathrm{d}, \mathrm{J}=17.9 \mathrm{~Hz}, 1 \mathrm{H}), 3.78(\mathrm{~d}, \mathrm{~J}=17.9 \mathrm{~Hz}, 1 \mathrm{H}), 3.64(\mathrm{~s}, 3 \mathrm{H}), 3.13(\mathrm{dd}, \mathrm{J}=18.7,10.6,1 \mathrm{H}), 2.62$ (dd, J = 18.7, $2.7 \mathrm{~Hz}, 1 \mathrm{H}) . \mathrm{MS}: m / z 278(\mathrm{M}+\mathrm{H})^{+}$

(2S)-1-Benzyl-2-methyl-4,4-difluoropyrrolidine-1,2-dicarboxylate (8). A solution of compound 99 (8.83 g, $31.85 \mathrm{mmol})$ in DCM $(6 \mathrm{ml})$ was slowly added to a solution of diethylaminosulfur fluoride (8.35 $\mathrm{ml}, 63.7 \mathrm{mmol})$ in DCM $(32 \mathrm{ml})$ precooled to $-78^{\circ} \mathrm{C}$. The reaction mixture was warmed to r.t. and mixed with cold water. The organic layer was separated, washed with water, dried $\left(\mathrm{Na}_{2} \mathrm{SO}_{4}\right)$ and evaporated to give title compound $\mathbf{8}$ as a yellow oil which was used in the next step without any further purification. ${ }^{1} \mathrm{H}$ NMR (DMSO $d 6,400$ MHz, 330K) $\delta$ 7.40-7.32 (m, 5H), 5.16-5.12 (m, 2H), 4.63 (bs, 1H), 3.96-3.80 (m, $2 \mathrm{H}), 3.65(\mathrm{~s}, 3 \mathrm{H}), 3.15-2.86(\mathrm{~m}, 1 \mathrm{H}), 2.56-2.45$ (partially under DMSO) (m, 1H). MS m/z $300(\mathrm{M}+\mathrm{H})^{+}$.

1-[(Benzyloxy)carbonyl]-4,4-difluoro-L-proline (9). A solution of compound 8 (2.08 g, $6.95 \mathrm{mmol})$ in methanol $(70 \mathrm{ml})$ was refluxed with $2 \mathrm{~N} \mathrm{NaOH}(6.95 \mathrm{ml}, 6.95 \mathrm{mmol})$ for $2 \mathrm{~h}$. Methanol was removed and $\mathrm{pH}$ adjusted to 1 with $3 \mathrm{~N} \mathrm{HCl}$ obtaining a suspension which was extracted several times with ethyl acetate. Combined organics were dried $\left(\mathrm{Na}_{2} \mathrm{SO}_{4}\right)$ and evaporated to give title product 9 as a dark brown oil (quantitative yield). ${ }^{1} \mathrm{H}$ NMR $\left(\mathrm{DMSO}_{d 6}, 400 \mathrm{MHz}, 330 \mathrm{~K}\right) \delta 12.96$ (bs, 1H), 7.36-7.31 (m, 5H), 5.11 (s, 2H), $4.50(\mathrm{bs}, 1 \mathrm{H}), 3.91-3.80(\mathrm{~m}, 2 \mathrm{H}), 3.01-2.82(\mathrm{~m}, 1 \mathrm{H}), 2.56-2.41$ (partially under DMSO) (m, 1H).

MS $m / z 284(\mathrm{M}-\mathrm{H})^{+}$.

Benzyl-(2S)-2-aminocarbonyl-4,4-difluoropyrrolidine-1-carboxylate (10). To a stirred solution of compound 9 (1.98 g, $6.95 \mathrm{mmol})$, pyridine $(0.36 \mathrm{ml})$ and $\mathrm{Boc}_{2} \mathrm{O}(1.97 \mathrm{~g}, 9.03 \mathrm{mmol})$ in dioxane $(10 \mathrm{ml})$, ammonium bicarbonate $(0.692 \mathrm{~g}, 8.76 \mathrm{mmol})$ was added and the mixture was stirred at r.t. for $20 \mathrm{~h}$. Dioxane was concentrated and the residue dissolved in ethyl acetate and washed with $\mathrm{HCl} 1 \mathrm{~N}, \mathrm{NaHCO}_{3}$ sat. sol. and brine, dried $\left(\mathrm{Na}_{2} \mathrm{SO}_{4}\right)$, filtered and evaporated in vacuo to obtain the title compound $\mathbf{1 0}$ as a 
yellow oil (quantitative yield). Two sets of signals, two conformers (ratio 1:1) were present. ${ }^{1} \mathrm{H}$ NMR $\left(\mathrm{DMSO}_{d 6}, 400 \mathrm{MHz}\right) \delta 7.56(\mathrm{~d}, \mathrm{~J}=15.4 \mathrm{~Hz}, 1 \mathrm{H}), 7.39-7.34(\mathrm{~m}, 5 \mathrm{H}), 7.17(\mathrm{~d}, \mathrm{~J}=19.3 \mathrm{~Hz}, 1 \mathrm{H}), 5.10-5.08$ $(\mathrm{m}, 2 \mathrm{H}), 4.42(\mathrm{dd}, \mathrm{J}=9.3,4.7,0.5 \mathrm{H}), 4.34(\mathrm{dd}, \mathrm{J}=9.2,4.6 \mathrm{~Hz}, 0.5 \mathrm{H}), 3.92-3.73(\mathrm{~m}, 2 \mathrm{H}), 2.90-2.72(\mathrm{~m}$, $1 \mathrm{H}), 2.43-2.30(\mathrm{~m}, 1 \mathrm{H})$. MS $m / z 285(\mathrm{M}+\mathrm{H})^{+}$.

Benzyl-(2S)-2-cyano-4,4-difluoropyrrolidine-1-carboxylate (81n). A solution of compound 10 (1.97 g, $6.93 \mathrm{mmol})$ and $\mathrm{Et}_{3} \mathrm{~N}(2.02 \mathrm{ml}, 14.55 \mathrm{mmol})$ in $\mathrm{DCM}(14 \mathrm{ml})$ was cooled to $0{ }^{\circ} \mathrm{C}$ and trifluoroacetic anhydride $(0.98 \mathrm{ml}, 7.62 \mathrm{mmol})$ was added dropwise under nitrogen. Stirring was continued for $1 \mathrm{~h}$ allowing the mixture to reach r.t.. Volatiles were removed in vacuo and the residue was taken up in ethyl acetate, washed with $\mathrm{HCl} 1 \mathrm{~N}$, brine and dried $\left(\mathrm{Na}_{2} \mathrm{SO}_{4}\right)$. Evaporation gave the title compound 81n as brown oil (quantitative yield). ${ }^{1} \mathrm{H}$ NMR $\left(\mathrm{DMSO}_{d 6}, 400 \mathrm{MHz}\right) \delta 7.40-7.34(\mathrm{~m}, 5 \mathrm{H}), 5.20-5.03(\mathrm{~m}, 3 \mathrm{H})$, 3.99-3.72 (m, 2H), 3.06-2.69 (m, 2H).

(2S)-Benzyl-2-[amino(hydroxyimino)methyl]-4,4-difluoropyrrolidine-1-carboxylate (82n). A solution of compound $81 \mathrm{n}(1.85 \mathrm{~g}, 6.95 \mathrm{mmol}), \mathrm{NH}_{2} \mathrm{OH} \cdot \mathrm{HCl}(0.679 \mathrm{~g}, 9.73 \mathrm{mmol})$ and $\mathrm{Et}_{3} \mathrm{~N}(1.65 \mathrm{ml}, 11.82$ mmol) in ethanol (19.4 ml) was refluxed under nitrogen for $5 \mathrm{~h}$. Mixture was concentrated and residues taken up in ethyl acetate and washed with water and brine. Combined organics were dried $\left(\mathrm{Na}_{2} \mathrm{SO}_{4}\right)$ and evaporated to give the title compound 82n (yield 88\%) as a foam. ${ }^{1} \mathrm{H}$ NMR $\left(\mathrm{DMSO}_{d 6}, 300 \mathrm{MHz}, 330 \mathrm{~K}\right.$ ) $\delta 9.12$ (bs, 1H), 7.38-7.34 (m, 5H), 5.36 (bs, 2H), 5.13 (d, J = $14.4 \mathrm{~Hz}, 1 \mathrm{H}), 5.09$ (d, J = 14.4 Hz, 1H), $4.56(\mathrm{dd}, \mathrm{J}=8.6,4.9 \mathrm{~Hz}, 1 \mathrm{H}), 4.07-3.76(\mathrm{~m}, 2 \mathrm{H}), 2.80-2.71(\mathrm{~m}, 1 \mathrm{H}), 2.60-2.51$ (partially under DMSO) (m, 1H). MS m/z $300(\mathrm{M}+\mathrm{H})^{+}$.

\section{Dimethyl-2-\{[(amino-\{(2S)-1-[(benzyloxy)carbonyl]-4,4-difluoropyrrolidin-2-yl\}-methylidene)-}

amino]-oxy\}-but-2-enedioate (83n). A solution of compound 82n (8.5 g, $28.2 \mathrm{mmol})$ and dimethylacetylendicarboxylate $(4.5 \mathrm{ml}, 36.9 \mathrm{mmol})$ in chloroform $(35 \mathrm{ml})$ was refluxed for $1 \mathrm{~h}$ under nitrogen and the solution was concentrated. Residue was purified by column chromatography $\left(\mathrm{SiO}_{2}\right.$, petroleum ether:ethyl acetate=7.5:2.5), to give the desired product 83n as a 3:1 mixture of two isomers ( yield 42\%). ${ }^{1} \mathrm{H}$ NMR $\left(\mathrm{DMSO}_{d 6}, 300 \mathrm{MHz}, 330 \mathrm{~K}\right) \delta$ 7.45-7.25 (m, 5H), 6.63 (bs, 1.5H), 6.30 (bs, 0.5H), $5.62(\mathrm{~s}, 0.75 \mathrm{H}), 5.60(\mathrm{~s}, 0.25 \mathrm{H}), 5.13(\mathrm{~s}, 2 \mathrm{H}), 4.58(\mathrm{dd}, \mathrm{J}=9.1,4.9 \mathrm{~Hz}), 4.57$ (dd, partially overlapped) $(1 \mathrm{H}), 3.96-3.86(\mathrm{~m}, 2 \mathrm{H}), 3.79(\mathrm{~s}, 2.2 \mathrm{H}), 3.74(\mathrm{~s}, 0.8 \mathrm{H}), 3.66(\mathrm{~s}, 0.8 \mathrm{H}), 3.61(\mathrm{~s}, 2.2 \mathrm{H}), 2.93-2.81(\mathrm{~m}, 1 \mathrm{H})$, 2.56-2.43 (partially under DMSO, $\mathrm{m}, 1 \mathrm{H})$. MS $m / z 442(\mathrm{M}+\mathrm{H})^{+}$.

(2S)-Methyl-2-\{1-[(benzyloxy)carbonyl]-4,4-difluoropyrrolidin-2-yl\}-5,6-dihydroxypyrimidine-4-

carboxylate (84n). A solution of compound 83n (4.6 g, $10.5 \mathrm{mmol})$ in $o$-xylenes $(120 \mathrm{ml})$ was refluxed for $6 \mathrm{~h}$. Then the reaction was cooled down and concentrated. Ethyl ether was added until precipitation of 
a solid that was filtered, washed with other ethyl ether and dried to give the title pyrimidine $\mathbf{8 4 n}$ as a brown solid (35\%). Two sets of signals, two rotamers (ratio 1:1) were present. ${ }^{1} \mathrm{H}$ NMR $\left(\mathrm{DMSO}_{d 6}, 400\right.$ $\mathrm{MHz}) \delta 12.97(\mathrm{~s}, 1 \mathrm{H}), 10.38(\mathrm{~s}, 1 \mathrm{H}), 7.40-7.29(\mathrm{~m}, 3 \mathrm{H}), 7.22-7.15(\mathrm{~m}, 1 \mathrm{H}), 7.10-7.05(\mathrm{~m}, 1 \mathrm{H}), 5.12(\mathrm{~d}, \mathrm{~J}=$ $12.6 \mathrm{~Hz}, 0.5 \mathrm{H}) 5.10(\mathrm{~s}, 1 \mathrm{H}), 4.89(\mathrm{~d}, \mathrm{~J}=12.6 \mathrm{~Hz}, 0.5 \mathrm{H}), 4.86-4.72(\mathrm{~m}, 1 \mathrm{H}), 4.10-3.86(\mathrm{~m}, 2 \mathrm{H}), 3.81(\mathrm{~s}$, $3 \mathrm{H}), 2.90-2.85(\mathrm{~m}, 1 \mathrm{H}), 2.64-2.53$ (partially under DMSO, m, 1H). MS $m / z 410(\mathrm{M}+\mathrm{H})^{+}$.

Methyl 5-(benzoyloxy)-2-\{(2S)-1-[(benzyloxy)carbonyl]-4,4-difluoropyrrolidin-2-yl\}-6hydroxypyrimidine-4-carboxylate (85n). Compound 84n (2.7 g, $6.7 \mathrm{mmol})$ in dry pyridine $(27 \mathrm{ml})$ was treated with benzoic anhydride $(3.03 \mathrm{~g}, 13.4 \mathrm{mmol})$ overnight at r.t.. The mixture was evaporated, taken up in ethyl acetate $(400 \mathrm{ml})$ and washed with $\mathrm{HCl} 1 \mathrm{~N}(2 \times 150 \mathrm{ml})$ and brine. Organics were dried $\left(\mathrm{Na}_{2} \mathrm{SO}_{4}\right)$, filtered and evaporated to obtain an oil (yield 33\%) which was purified by column chromatography $\left(\mathrm{SiO}_{2}\right.$, ethyl acetate:petroleum ether=7:3) to give 85n. ${ }^{1} \mathrm{H}$ NMR $\left(\mathrm{DMSO}_{d 6}, 300 \mathrm{MHz}, 330\right.$ K) $\delta 13.51(\mathrm{bs}, 1 \mathrm{H}), 8.10(\mathrm{~d}, \mathrm{~J}=7.6 \mathrm{~Hz}, 2 \mathrm{H}), 7.79(\mathrm{t}, \mathrm{J}=7.1 \mathrm{~Hz}, 1 \mathrm{H}), 7.64(\mathrm{t}, \mathrm{J}=7.6 \mathrm{~Hz}, 2 \mathrm{H}), 7.33-7.17$ $(\mathrm{m}, 5 \mathrm{H}), 5.13(\mathrm{~s}, 2 \mathrm{H}), 4.99(\mathrm{t}, \mathrm{J}=7.3 \mathrm{~Hz}, 1 \mathrm{H}), 4.09-3.97(\mathrm{~m}, 2 \mathrm{H}), 3.77(\mathrm{~s}, 3 \mathrm{H}), 3.02-2.99(\mathrm{~m}, 2 \mathrm{H})$. MS: $m / z 514(\mathrm{M}+\mathrm{H})^{+}$.

Methyl-5-(benzoyloxy)-2-\{(2S)-1-[(benzyloxy)carbonyl]-4,4-difluoropyrrolidin-2-yl\}-1-methyl-6-oxo1,6-dihydropyrimidine-4-carboxylate (86n). Compound 85n (826 mg, $1.61 \mathrm{mmol})$ dissolved in dry dioxane $(15 \mathrm{ml})$ was added to a suspension of $\mathrm{LiH}(15.35 \mathrm{mg}, 1.93 \mathrm{mmol})$ in dioxane $(11 \mathrm{ml})$. The mixture was stirred at $38^{\circ} \mathrm{C}$ for 45 minutes and then cooled down to r.t.. Dimethyl sulphate (0.199 $\left.\mathrm{ml}, 2.09 \mathrm{mmol}\right)$ was added and the mixture was warmed to $58{ }^{\circ} \mathrm{C}$ for $1 \mathrm{~h}$. The reaction mixture was cooled down to $16^{\circ} \mathrm{C}$ and glacial acetic acid $(2 \mathrm{ml})$ was added, followed by water $(1 \mathrm{ml})$ and ethyl acetate $(100 \mathrm{ml})$. The combined organic layers were dried $\left(\mathrm{Na}_{2} \mathrm{SO}_{4}\right)$, filtered and concentrated to an oil which was cromatographed $\left(\mathrm{SiO}_{2}\right.$, ethyl acetate:petroleum ether=3:7) to give the desired compound 86n as a 1:1 mixture of two rotamers, (yield 73\%). ${ }^{1} \mathrm{H}$ NMR $\left(\mathrm{DMSO}_{d 6}, 300 \mathrm{MHz}\right) \delta 8.11-8.08(\mathrm{~m}, 2 \mathrm{H}), 7.80(\mathrm{t}, \mathrm{J}=7.7$ $\mathrm{Hz}, 1 \mathrm{H}), 7.67-7.65(\mathrm{~m}, 2 \mathrm{H}), 7.36-7.10(\mathrm{~m}, 5 \mathrm{H}), 5.50(\mathrm{dd}, \mathrm{J}=9.2,4.7 \mathrm{~Hz}, 1 \mathrm{H}), 5.22(\mathrm{~d}, \mathrm{~J}=12.9 \mathrm{~Hz}, 0.5 \mathrm{H})$, 5.14-4.95 (m, 1H), $4.93(\mathrm{~d}, \mathrm{~J}=12.3 \mathrm{~Hz}, 0.5 \mathrm{H}), 4.16-3.79(\mathrm{~m}, 2 \mathrm{H}), 3.74(\mathrm{~s}, 3 \mathrm{H}), 3.61(\mathrm{~s}, 1.5 \mathrm{H}), 3.45(\mathrm{~s}$, $1.5 \mathrm{H}), 3.25-3.11(\mathrm{~m}, 1 \mathrm{H}), 2.89-2.74(\mathrm{~m}, 1 \mathrm{H}) . \mathrm{MS}: m / z 528(\mathrm{M}+\mathrm{H})^{+}$.

(2S)-Benzyl-4,4-difluoro-2-(4-\{[(4-fluorobenzyl)amino]carbonyl\}-5-hydroxy-1-methyl-6-oxo-1,6dihydropyrimidin-2-yl)pyrrolidine-1-carboxylate (89n). Compound 86n (133 mg, $0.25 \mathrm{mmol})$ in dry $\mathrm{MeOH}(2.5 \mathrm{ml})$ was treated with 4-fluorobenzyl amine $(0.071 \mathrm{ml}, 0.625 \mathrm{mmol})$ at reflux for $2 \mathrm{~h}$. Solvent was removed in vacuo and the residue was taken up in ethyl acetate $(50 \mathrm{ml})$, washed with $\mathrm{HCl} 1 \mathrm{~N}(2 \times 30$ $\mathrm{ml})$, brine, dried $\left(\mathrm{Na}_{2} \mathrm{SO}_{4}\right)$. The filtrate was concentrated in vacuo and triturated with ethyl ether to obtain 
the title compound 89n as a 1.5:1 mixture of two rotamers by NMR (78\% yield). ${ }^{1} \mathrm{H}$ NMR $\left(\mathrm{DMSO}_{d 6}\right.$ +TFA, $300 \mathrm{MHz}) \delta 8.92$ (bt, 0.4H), 8.69 (bt, 0.6H), 7.36-7.31 (m, 4H), 7.20-7.09 (m, 4H), 6.97 (d, J = 7.2 $\mathrm{Hz}, 1 \mathrm{H}), 5.34-5.25(\mathrm{~m}, 1 \mathrm{H}), 5.14(\mathrm{~d}, \mathrm{~J}=12.4 \mathrm{~Hz}, 0.4 \mathrm{H}), 5.07-4.99(\mathrm{~m}, 1.2 \mathrm{H}), 4.81(\mathrm{~d}, \mathrm{~J}=12.2 \mathrm{~Hz}, 0.4 \mathrm{H})$, 4.51-4.48 (m, 2H), 4.38-4.21 (m, 1H), 4.07-3.96 (m, 1H), 3.59 (s, 1.2H), $3.48(\mathrm{~s}, 1.8 \mathrm{H}), 3.05-2.95(\mathrm{~m}$, $1 \mathrm{H}), 2.78-2.68(\mathrm{~m}, 1 \mathrm{H}) . \mathrm{MS} m / z 517(\mathrm{M}+\mathrm{H})^{+}$.

\section{(2S)-2-[4,4-difluoropyrrolidin-2-yl]- $N$-(4-fluorobenzyl)-5-hydroxy-1-methyl-6-oxo-1,6-}

dihydropyrimidine-4-carboxamide (90n). A solution of compound 89n (65 mg, $0.13 \mathrm{mmol}$ ) in $\mathrm{MeOH}$ (1.5 ml) was treated with $\mathrm{Pd} / \mathrm{C} 10 \%(6.5 \mathrm{mg})$ for $3 \mathrm{~h}$ at r.t. under $\mathrm{H}_{2}$ atmosphere. The mixture was filtrated over a celite pad, concentrated in vacuo to give a pale yellow residue 90n that was used without further purification (96\% yield). ${ }^{1} \mathrm{H}$ NMR $\left(\mathrm{DMSO}_{d \sigma}+\mathrm{TFA}, 300 \mathrm{MHz}, 340 \mathrm{~K}\right) \delta 9.60(\mathrm{bt}, 1 \mathrm{H}), 7.39(\mathrm{t}, \mathrm{J}=8 \mathrm{~Hz}$, 2H), $7.17(\mathrm{t}, \mathrm{J}=8.8 \mathrm{~Hz}, 2 \mathrm{H}), 5.35(\mathrm{t}, \mathrm{J}=8.4 \mathrm{~Hz}, 1 \mathrm{H}), 4.62(\mathrm{dd}, \mathrm{J}=15.3,6.6 \mathrm{~Hz}, 1 \mathrm{H}), 4.55(\mathrm{dd}, \mathrm{J}=15.2$, $6.3 \mathrm{~Hz}, 1 \mathrm{H}), 4.05-3.87(\mathrm{~m}, 2 \mathrm{H}), 3.48(\mathrm{~s}, 3 \mathrm{H}), 3.30-3.14(\mathrm{~m}, 1 \mathrm{H}), 2.96-2.78(\mathrm{~m}, 1 \mathrm{H}) . \mathrm{MS} m / z 383(\mathrm{M}+\mathrm{H})^{+}$. Scheme 6. ${ }^{a}$ Synthesis of compound $\mathbf{8 6 m}$.

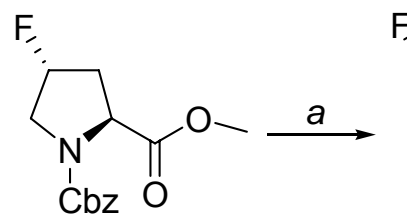

11<smiles>CC(=O)N1C[C@H](F)C[C@H]1C(N)=O</smiles>

12<smiles>N#CC1C[C@H](F)CN1C(=O)OCc1ccccc1</smiles>

$81 \mathrm{~m}$<smiles>CC1CCCCC1/C(N)=N/O</smiles>

$82 \mathrm{~m}$

F,

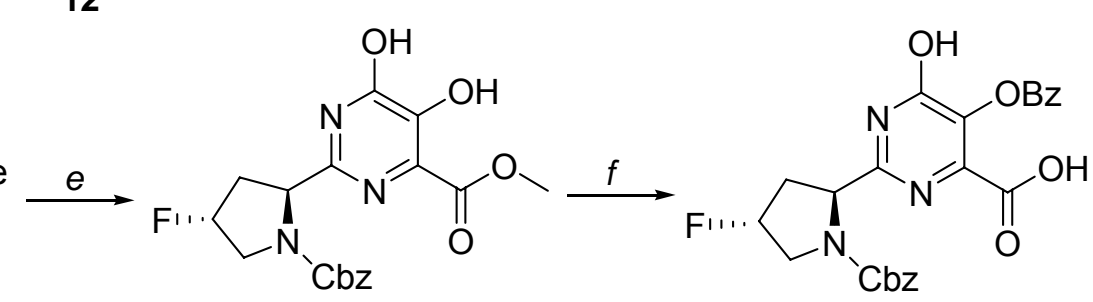

$83 \mathrm{~m}$<smiles>Cn1c([C@@H]2C[C@H](F)CN2C(=O)OCc2ccccc2)nc(C(=O)O)c(C(=O)OC(C)(C)C)c1=O</smiles>

$84 \mathrm{~m}$

$85 \mathrm{~m}$

$86 \mathrm{~m}$

${ }^{a}$ Reagents and conditions: (a) $\mathrm{NH}_{3} 32 \%$, THF, $40-80{ }^{\circ} \mathrm{C}$; (b) TFAA, Et 3 N, DCM; (c) $\mathrm{NH}_{2} \mathrm{OH} \cdot \mathrm{HCl}, \mathrm{Et}_{3} \mathrm{~N}$, EtOH, reflux, 5h; (d) DMAD, $\mathrm{CHCl}_{3}$, reflux, 1h; (e) $o$-xylenes, $158^{\circ} \mathrm{C}$; (f) $\mathrm{Bz} 2 \mathrm{O}, \mathrm{Py}, 3 \mathrm{~h}$; (g) LiH, dioxane, $\mathrm{Me}_{2} \mathrm{SO}_{4}$. 
(2S, 4R)-Benzyl 2-(aminocarbonyl)-4-fluoropyrrolidine-1-carboxylate (12). To compound 11 prepared according a literature procedure ${ }^{2}(4.4 \mathrm{~g}, 15.66 \mathrm{mmol})$ dissolved in THF $(4 \mathrm{~mL})$ in a closed vessel, ammonia (32 wt. \% solution in water, $40 \mathrm{~mL}$.) was added and the mixture was heated overnight at $40{ }^{\circ} \mathrm{C}$ and then $1 \mathrm{~h}$ at $80{ }^{\circ} \mathrm{C}$. Volatiles were evaporated affording the desired compound in quantitative yield (4.17 g). ${ }^{1} \mathrm{H}$ NMR $\left(\mathrm{DMSO}_{d 6}, 300 \mathrm{MHz}\right) \delta 7.48(\mathrm{~d}, \mathrm{~J}=10 \mathrm{~Hz}, 1 \mathrm{H}), 7.38-7.21(\mathrm{~m}, 5 \mathrm{H}), 7.02(\mathrm{~d}, \mathrm{~J}=20 \mathrm{~Hz}$, $1 \mathrm{H}), 5.24(\mathrm{~d}, \mathrm{~J}=39 \mathrm{~Hz}, 1 \mathrm{H}), 5.09-4.93(\mathrm{~m}, 2 \mathrm{H}), 4.32-4.09(\mathrm{~m}, 1 \mathrm{H}), 3.69-3.42$ (m, $2 \mathrm{H}), 2.30-2.52$ (partially obscured by DMSO, 1H), 2.15-1.89 (m, 1H).

Dimethyl-(2E and 2Z)-2-\{[(amino\{(2S,4R)-1-[(benzyloxy)carbonyl]-4-fluoropyrrolidin-2yl\}methylene)amino]oxy\}but-2-enedioate (83m). The primary amide 12 (4.167 g, $15.67 \mathrm{mmol})$ was dissolved in DCM (103 ml) and treated with $\mathrm{Et}_{3} \mathrm{~N}\left(4.6 \mathrm{ml}, 2.1\right.$ eq.). The mixture was cooled down to $0^{\circ} \mathrm{C}$ and trifluoroacetic anhydride $(2.43 \mathrm{ml}, 1.1$ eq.) was added. After $1 \mathrm{~h}$, volatiles were evaporated and the residue dissolved in EtOAc, washed with $\mathrm{HCl} 1 \mathrm{~N}, \mathrm{NaHCO}_{3}$ sat. sol. and brine, dried $\left(\mathrm{Na}_{2} \mathrm{SO}_{4}\right)$, filtered and evaporated in vacuo affording the desired nitrile $\mathbf{8 1 m}$ that was submitted to the next step without purification. To the nitrile $\mathbf{8 1 m}$ dissolved in ethanol $(41 \mathrm{ml}), \mathrm{NH}_{2} \mathrm{OH} \cdot \mathrm{HCl}(4.97 \mathrm{~g}, 1.3 \mathrm{eq}$.$) and \mathrm{Et}_{3} \mathrm{~N}(3.27$ $\mathrm{ml}, 1.5$ eq.) were added. The mixture was stirred at $40{ }^{\circ} \mathrm{C}$ overnight. The mixture was concentrated in vacuo and the residue dissolved in ethyl acetate washed with brine, dried $\left(\mathrm{Na}_{2} \mathrm{SO}_{4}\right)$, filtered and concentrated affording the desired compound $\mathbf{8 2} \mathbf{m}$ that was submitted to the next step without purification. MS m/z $249(\mathrm{M}+\mathrm{H})^{+}$. To compound 82m dissolved in chloroform (90 ml), dimethyl acetylendicarboxylate $(2.12 \mathrm{ml}, 1.1$ eq.) was added. The mixture was refluxed for $5 \mathrm{~h}$ and left stirring at 40 ${ }^{\circ} \mathrm{C}$ overnight. The chloroform was removed in vacuo and the crude was purified by column chromatography $\left(\mathrm{SiO}_{2}\right.$, petroleum ether:ethyl acetate=1:1 as ) yielding the title compound $\mathbf{8 3 m}$ as a 7:3 mixture of isomers $\left(2.22 \mathrm{~g}, 34 \%\right.$ yield over three steps). ${ }^{1} \mathrm{H}$ NMR $\left(\mathrm{DMSO}_{d 6}, 300 \mathrm{MHz}\right) \delta 7.47-7.21$ (m, 3.5H), 6.84-6.56 (m, 1.5H), 6.7-6.55 (bs, $2 \mathrm{H}), 5.63(\mathrm{~s}, 1 \mathrm{H}), 5.32(\mathrm{~d}, \mathrm{~J}=51.7 \mathrm{~Hz}, 1 \mathrm{H}), 5.18-4.99(\mathrm{~m}, 2 \mathrm{H})$, 4.44-4.21 (m, 1H), 3.94-3.48 (m, 8H), 2.67-2.37 (partialy obscured by DMSO, 1H), 2.34-2.03 (m, 1H).

\section{Methyl 5-(benzoyloxy)-2-\{(2S,4R)-1-[(benzyloxy)carbonyl]-4-fluoropyrrolidin-2-yl\}-6-} hydroxypyrimidine-4-carboxylate (85m). Compound 83m (1 g, $2.36 \mathrm{mmol})$ was dissolved in xylene (11 $\mathrm{ml}$ ) and the solution stirred at $150{ }^{\circ} \mathrm{C}$ for $7 \mathrm{~h}$ and at r.t. overnight. Xylene was concentrated in vacuo. To the crude compound $\mathbf{8 4 m}$, dissolved in pyridine $(11 \mathrm{ml})$, benzoic anhydride $(0.69 \mathrm{~g}, 1.3 \mathrm{eq}$.) was added, the reaction mixture was stirred at r.t. overnight. The solution was concentrated in vacuo and the crude dissolved in ethyl acetate washed with $\mathrm{HCl}(1 \mathrm{~N})$, and brine, dried $\left(\mathrm{Na}_{2} \mathrm{SO}_{4}\right)$, filtered and evaporated in vacuo. After purification by column chromatography $\left(\mathrm{SiO}_{2}\right.$, petroleum ether:ethyl acetate=55:45) the title 
compound 85m $\left(0.56,47 \%\right.$ yield) was obtained. ${ }^{1} \mathrm{H}$ NMR $\left(\mathrm{DMSO}_{d 6}, 400 \mathrm{MHz}, 330 \mathrm{~K}\right) \delta 13.39(\mathrm{bs}, 1 \mathrm{H})$, $8.08(\mathrm{~d}, \mathrm{~J}=7.4 \mathrm{~Hz}, 2 \mathrm{H}), 7.78(\mathrm{t}, \mathrm{J}=7.4 \mathrm{~Hz}, 1 \mathrm{H}), 7.63(\mathrm{t}, \mathrm{J}=7.6 \mathrm{~Hz}, 2 \mathrm{H}), 7.43-7.01(\mathrm{~m}, 5 \mathrm{H}), 5.41(\mathrm{~d}, \mathrm{~J}=$ $53.0 \mathrm{~Hz}, 1 \mathrm{H}), 5.21-4.83(\mathrm{~m}, 2 \mathrm{H}), 4.81(\mathrm{t}, \mathrm{J}=8.3 \mathrm{~Hz}, 1 \mathrm{H}), 3.96-3.77(\mathrm{~m}, 2 \mathrm{H}), 3.75(\mathrm{~s}, 3 \mathrm{H}), 2.75-2.59$ (partially under DMSO, m, 1H), 2.34-2.03 (m, 1H). MS m/z $496(\mathrm{M}+\mathrm{H})^{+}$.

Methyl 5-(benzoyloxy)-2-\{(2S,4R)-1-[(benzyloxy)carbonyl]-4-fluoropyrrolidin-2-yl\}-1-methyl-6-oxo1,6-dihydropyrimidine-4-carboxylate $\mathbf{( 8 6 m )}$. To compound $\mathbf{8 5 m}(0.56,1.12 \mathrm{mmol})$, dissolved in dioxane (12 ml), LiH (13 mg, 1.4 eq.) was added and the reaction mixture stirred at r.t. for $1 \mathrm{~h}$. The temperature was raised to $60{ }^{\circ} \mathrm{C}$ and dimethyl sulphate $(0.139 \mathrm{ml}, 1.3$ eq.) was added dropwise. After 12 $\mathrm{h}$, the reaction mixture was cooled down to $0{ }^{\circ} \mathrm{C}$ and $\mathrm{HCl} 1 \mathrm{~N}$ was added to quench the reaction. The reaction mixture was extracted with ethyl acetate and the organic phase washed with $\mathrm{HCl} 1 \mathrm{~N}$, and brine, dried $\left(\mathrm{Na}_{2} \mathrm{SO}_{4}\right)$, filtered and concentrated in vacuo. The title product $\mathbf{8 6 m}$ was isolated by column chromatography $\left(\mathrm{SiO}_{2}\right.$, petroleum ether:ethyl acetate=7:3) (0.48 g, 84\% yield). ${ }^{1} \mathrm{H} \mathrm{NMR}\left(\mathrm{DMSO}_{d 6}, 400\right.$ $\mathrm{MHz}, 330 \mathrm{~K}) \delta 8.08(\mathrm{~d}, \mathrm{~J}=7.4 \mathrm{~Hz}, 2 \mathrm{H}), 7.78(\mathrm{t}, \mathrm{J}=7.4 \mathrm{~Hz}, 1 \mathrm{H}), 7.63(\mathrm{t}, \mathrm{J}=7.4 \mathrm{~Hz}, 2 \mathrm{H}), 7.43-7.01(\mathrm{~m}$, $5 \mathrm{H}), 5.46(\mathrm{~d}, \mathrm{~J}=52.4 \mathrm{~Hz}, 1 \mathrm{H}), 5.30(\mathrm{t}, \mathrm{J}=7.6 \mathrm{~Hz}, 1 \mathrm{H}), 5.19-4.81(\mathrm{~m}, 2 \mathrm{H}), 3.98-3.56(\mathrm{~m}, 7 \mathrm{H}), 3.54-3.41$ (m, 1H), 2.90-2.70 (m, 1H), 2.46-2.30 (partially under DMSO, m, $1 \mathrm{H}$ ).

Scheme 7. ${ }^{a}$ Synthesis of compound $85 b$ and $85 e$.<smiles>[R]C1CC([R])N(C(=O)OCc2ccccc2)C1[N]</smiles>

81b,e

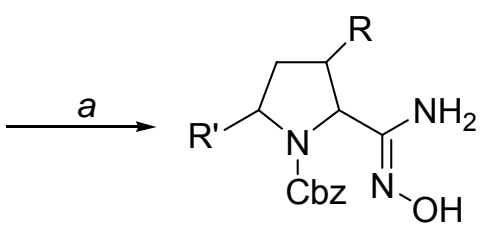

82b,e

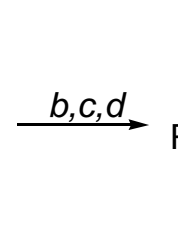

$85 b, e$

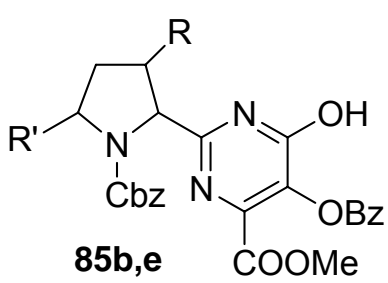

b $\mathrm{R}=\mathrm{Me} \mathrm{R}^{\prime}=\mathrm{H}$ e $R=H \quad R^{\prime}=M e$

${ }^{a}$ Reagents and conditions: (a) $\mathrm{NH}_{2} \mathrm{OH}, \mathrm{MeOH}$; (b) DMAD, $\mathrm{CHCl}_{3}$, reflux; (c) xylenes, reflux; (d) $\mathrm{Bz}_{2} \mathrm{O}$, Pyridine.

Benzyl 2-[(Z)-amino(hydroxyimino)methyl]-3-methylpyrrolidine-1-carboxylate (82b). A solution of hydroxylamine hydrochloride $(0.57 \mathrm{~g}, 8.2 \mathrm{mmol})$ in $\mathrm{MeOH}(10 \mathrm{~mL})$ was added at $0{ }^{\circ} \mathrm{C}$ to a solution of $\mathrm{KOH}(0.46 \mathrm{~g}, 8.2 \mathrm{mmol})$ in $\mathrm{MeOH}(10 \mathrm{~mL})$. The resulting reaction mixture was filtered and added to a solution of nitrile $\mathbf{8 1 b}(2.0 \mathrm{~g}, 8.2 \mathrm{mmol})$ in $\mathrm{MeOH}(20 \mathrm{~mL})$ and stirred at $40{ }^{\circ} \mathrm{C}$ for $4 \mathrm{~h}$ until the starting material was consumed. The solvent was removed in vacuo and the residue was taken up in $\mathrm{CHCl}_{3}$, washed with water, brine and dried $\left(\mathrm{Na}_{2} \mathrm{SO}_{4}\right)$. Evaporation of solvent gave a residue 82b which was used in the next step without further purification. ${ }^{1} \mathrm{H}$ NMR $\left(\mathrm{CDCl}_{3}, 300 \mathrm{MHz}\right) \delta 7.40-7.30(\mathrm{~m}, 5 \mathrm{H}), 5.20-5.10$ (m, 2H), 4.70-4.50 (m, 1H), 3.70-3.60 (m, 1H), 3.50-3.30 (m, 1H), 2.50-2.30 (m, 1H), 2.00-1.80 (m, 1H), $1.70-1.50(\mathrm{~m}, 1 \mathrm{H}), 1.10-0.90(\mathrm{~m}, 3 \mathrm{H})$. 
Benzyl 2-[(Z)-amino(hydroxyimino)methyl]-5-methylpyrrolidine-1-carboxylate (82e). Following the same reaction described for compound 82b, compound 82e was obtained from nitrile 81e. ${ }^{1} \mathrm{H}$ NMR $\left(\mathrm{CDCl}_{3}, 300 \mathrm{MHz}\right) \delta$ 7.40-7.30 (m, 5H), 5.20-5.00 (m, 2H), 4.60-4.40 (m, 1H), 4.20-4.00 (m, 1H), 2.30$1.90(\mathrm{~m}, 3 \mathrm{H}), 1.70-1.50(\mathrm{~m}, 1 \mathrm{H}), 1.30-1.10(\mathrm{~m}, 3 \mathrm{H})$.

Methyl 5-(benzoyloxy)-2-\{1-[(benzyloxy)carbonyl]-3-methylpyrrolidin-2-yl\}-6-hydroxypyrimidine4-carboxylate (85b). A solution of compound $\mathbf{8 2 b}$ (2.8 g, $10.1 \mathrm{mmol})$ in $\mathrm{CHCl}_{3}$ was treated with dimethyl acetylene dicarboxylate $(1.3 \mathrm{~mL}, 13.1 \mathrm{mmol})$ and refluxed for $3 \mathrm{~h}$. The reaction mixture was cooled to r.t. and concentrated. The residue $\mathbf{8 3 b}$ was taken in xylene and refluxed for $24 \mathrm{~h}$. The solvent was removed in vacuo and the crude residue $\mathbf{8 4 b}$ was taken in pyridine $(10 \mathrm{~mL})$. To this solution, benzoic anhydride $(2.4$ g, $10.5 \mathrm{mmol}$ ) was added and the reaction mixture was stirred at r.t. until the starting material was consumed. The reaction mixture was concentrated and the oily residue was diluted with EtOAc and washed with $1 \mathrm{~N} \mathrm{HCl}, \mathrm{NaHCO}_{3}$ sat. sol. and brine. The organic phase was dried $\left(\mathrm{Na}_{2} \mathrm{SO}_{4}\right)$, filtered and concentrated and the residue was purified by column chromatography $\left(\mathrm{SiO}_{2}\right.$, Petrol. Ether:EtOAc=1:1) to obtain the title compound $\mathbf{8 5 b}$ as a mixture of diastereoisomers, $22 \%$. ${ }^{1} \mathrm{H}-\mathrm{NMR}\left(\mathrm{CDCl}_{3}, 300 \mathrm{MHz}\right) \delta$ : 11.98 (bs, 1H), 8.02 (d, J = 7.5 Hz, 2H), 7.50 (t, J = 7.3 Hz, 1H), 7.35 (t, J = 7.5 Hz, 2H), 7.20-7.00 (m, $5 \mathrm{H}), 5.10-4.70(\mathrm{~m}, 3 \mathrm{H}), 3.70(\mathrm{~s}, 2 \mathrm{H}), 3.68(\mathrm{~s}, 1 \mathrm{H}), 3.50-3.30(\mathrm{~m}, 1 \mathrm{H}), 3.20-2.80(\mathrm{~m}, 1 \mathrm{H}), 2.60-2.40(\mathrm{~m}$, $1 \mathrm{H}), 1.70-1.50(\mathrm{~m}, 1 \mathrm{H}), 1.00(\mathrm{~d}, \mathrm{~J}=6.6 \mathrm{~Hz}, 1 \mathrm{H}), 0.77(\mathrm{~d}, \mathrm{~J}=6.6 \mathrm{~Hz}, 2 \mathrm{H})$.

Methyl 5-(benzoyloxy)-2-\{1-[(benzyloxy)carbonyl]-5-methylpyrrolidin-2-yl\}-6-hydroxypyrimidine4-carboxylate (85e). Following the same three steps described to obtained 85b, compound 85e was obtained from 82e. ${ }^{1} \mathrm{H}-\mathrm{NMR}\left(\mathrm{CDCl}_{3}, 300 \mathrm{MHz}\right) \delta 8.06(\mathrm{~d}, \mathrm{~J}=8.0 \mathrm{~Hz}, 2 \mathrm{H}), 7.74(\mathrm{t}, \mathrm{J}=7.0 \mathrm{~Hz}, 1 \mathrm{H}), 7.60$ $(\mathrm{t}, \mathrm{J}=8.0 \mathrm{~Hz}, 2 \mathrm{H}), 7.40-7.30(\mathrm{~m}, 5 \mathrm{H}), 5.10-5.00(\mathrm{~m}, 3 \mathrm{H}), 4.30-4.20(\mathrm{~m}, 1 \mathrm{H}), 3.71(\mathrm{~s}, 2 \mathrm{H}), 3.70(\mathrm{~s}, 1 \mathrm{H})$, 2.30-2.20 (m, 2H), 1.80-1.50 (m, 2H), $1.40(\mathrm{~d}, \mathrm{~J}=6.4 \mathrm{~Hz}, 1 \mathrm{H}), 1.21(\mathrm{~d}, \mathrm{~J}=6.4 \mathrm{~Hz}, 2 \mathrm{H})$.

\section{1-tert-Butyl (2S)-2-[(Z)-amino(hydroxyimino)methyl]-4-methylpyrrolidine-1-carboxylate (82c,d)}

A solution of $\mathrm{NH}_{2} \mathrm{OH} \cdot \mathrm{HCl}(0.7 \mathrm{~g}, 10.1 \mathrm{mmol})$ in $\mathrm{MeOH}(10 \mathrm{~mL})$ was added at $0{ }^{\circ} \mathrm{C}$ to a solution of $\mathrm{KOH}$ $(0.57 \mathrm{~g}, 10.1 \mathrm{mmol})$ in $\mathrm{MeOH}(10 \mathrm{~mL})$. The resulting reaction mixture was filtered and added to a solution of nitriles 81c and 81d $(2.12 \mathrm{~g}, 10.1 \mathrm{mmol})$ in $\mathrm{MeOH}(20 \mathrm{~mL})$ and stirred at $40{ }^{\circ} \mathrm{C}$ for $4 \mathrm{~h}$ until the starting material was consumed. The solvent was removed in vacuo and the residue was taken up in $\mathrm{CHCl}_{3}$, washed with water, brine and dried $\left(\mathrm{Na}_{2} \mathrm{SO}_{4}\right)$. Evaporation of solvent gave compounds 82c and 82d which were used in the next step without further purification. ${ }^{1} \mathrm{H}$ NMR $\left(\mathrm{DMSO}_{d 6}, 300 \mathrm{MHz}\right) \delta 5.21(\mathrm{bs}, 1 \mathrm{H})$, 4.20-3.90 (m, 1H), 3.70-3.50 (m, 1H), 2.90-2.80 (m, 1H), 2.20-2.10 (m, 1H), 2.10-2.00 (m, 1H), 1.37, 1.34 (2s, 9H), $0.98(\mathrm{~d}, \mathrm{~J}=6.5 \mathrm{~Hz}, 3 \mathrm{H})$. 


\section{PHARMACOKINETICS IN LABORATORY ANIMALS (in vivo studies) \\ MATERIALS AND METHODS}

In Vivo Studies

1. Rats

Male Sprague-Dawley rats (250-350 g), were used for the absorption disposition studies. In each rat, an indwelling cannula was implanted in the right jugular vein for blood sampling. The surgery was performed under light anaesthesia (Ketamine-Xilazine ( $85 \mathrm{mg} / \mathrm{kg}$ and $2.5 \mathrm{mg} / \mathrm{kg}$ respectively i.m.) one day prior the experiment. During the kinetic study, all animals were housed individually in plastic metabolism cages, and were unrestrained throughout the experiment. Compounds were dissolved in $20 \%$ DMSO/60\% PEG400/20\% Water for intravenous administration, and dissolved or suspended in 1\% Methylcellulose for oral administration. After an overnight fast, the rats received an i.v. (via caudal vein) or an oral dose of compound. Blood samples were collected at different times point after dosing. Plasma was separated immediately after blood sampling by centrifugation, and the plasma samples were kept frozen $\left(-20^{\circ} \mathrm{C}\right)$ until assayed by LC/MS/MS.

\section{Dogs}

Six male beagle dogs (three for each route of administration), weighing 4-9 $\mathrm{kg}$, were used in the absorption and kinetics studies. After an overnight fast, compounds were given iv or orally. Blood samples were collected at different times point after dosing. Plasma was separated immediately after blood sampling by centrifugation, and the plasma samples were kept frozen $\left(-20^{\circ} \mathrm{C}\right)$ until assayed by LC/MS/MS. Compounds were dissolved in 20\% DMSO/60\% PEG400/20\% Water for intravenous administration, and dissolved or suspended in 1\% Methylcellulose for oral administration. All animals were housed individually in metabolism cages. All samples were kept frozen $\left(-20^{\circ} \mathrm{C}\right)$ until assayed by LC/MS/MS.

\section{Monkeys}

Male rhesus monkeys, weighing 4-7 kg, were used in the absorption and kinetics studies. These studies were performed with a washout period at least one week between doses. After an overnight fast, compound was given iv (in DMSO) or orally (0.5\% Methylcellulose). Blood samples were collected at different times point after dosing. Plasma was separated immediately after blood sampling by centrifugation, and the plasma samples were kept frozen $\left(-20^{\circ} \mathrm{C}\right)$ until assayed by LC/MS/MS.

\section{Analytical Procedures}


Plasma samples were extracted using Liquid Handling Robot MultiProbe Packard by protein precipitation with acetonitrile. Then the samples were centrifuged $\left(3000 \mathrm{rpm} \times 15 \mathrm{~min}\right.$. at $\left.4^{\circ} \mathrm{C}\right)$ and the supernatant transferred and dried under nitrogen. The samples were reconstituted in Water/Acetonitrile 90/10 and then injected directly into an HPLC column. Sample analyses were performed using an API 3000 or/and API 2000 or/and API 4000 Mass Spectrometer interfaced via the Turbo Ion Spray (ESI)/APCI to a LC system consisting of a HTS PAL CTC autosampler and an Agilent HP 1100 Binary Pump. The results are calculated using Analyst Software linear regression with $1 /\left(x^{*} x\right)$ weighting. The Assay Precision was calculated for the Quality Controls by Watson Lims database.

Pharmacokinetic Analysis

The plasma clearance (CLp) of compounds were calculated (using Watson PK program) as the dose divided by the area under the plasma concentration-time curve from time zero to infinity (AUC0- $\infty$ ). The apparent half-life was estimated from the slope of the terminal phase of the log plasma concentration-time data. The volume of distribution (Vdss) was determined using the following noncompartmental method: $\mathrm{Vdss}=($ Dose IV $\mathrm{x}$ AUMC $) /(\mathrm{AUC} 0-\infty) 2$ where $\mathrm{AUMC}$ is the total area under the first moment of the drug concentrationtime curve from time zero to infinity. Bioavailability was estimated as the AUC0- $\infty$ ratio following oral and intravenous administration, normalized for differences in dose.

\section{Accurate mass measurements}

The accurate mass measurements were carried out on a chromatographic time scale by injecting $5 \mu \mathrm{L}$ of each working solution on a Waters $\mathrm{C}_{18}$ Sunfire $(20 \times 2.1 \mathrm{~mm}, 5 \mu \mathrm{m})$ column using an isocratic elution (Solvent A:Solvent B, 50:50) of Solvent A: water $+0.1 \%$ formic acid and Solvent B: acetonitrile $+0.1 \%$ formic acid at a flow rate of $1000 \mu \mathrm{L} / \mathrm{min}$.

The accurate mass measurements were performed in Selected Ion Monitoring (SIM) scan using reference lock masses. In particular for the present AM measurements a calibration mixture of $15 \mu \mathrm{g} / \mathrm{mL}$ Imazalil $\left([\mathrm{M}+\mathrm{H}]^{+}, \mathrm{C}_{14} \mathrm{H}_{15} \mathrm{Cl}_{2} \mathrm{~N}_{2} \mathrm{O}, 297.0561\right), 10 \mu \mathrm{g} / \mathrm{mL}$ Cinnarizine ([M+H] $\left.]^{+}, \mathrm{C}_{26} \mathrm{H}_{29} \mathrm{~N}_{2}, 369.2331\right), 50 \mu \mathrm{g} / \mathrm{mL}$

Ketoconazole $\left([\mathrm{M}+\mathrm{H}]^{+}, \quad \mathrm{C}_{26} \mathrm{H}_{29} \mathrm{Cl}_{2} \mathrm{~N}_{4} \mathrm{O}_{4}, \quad 531.1566\right)$ and $100 \mu \mathrm{g} / \mathrm{mL} \quad$ Itraconazole $\left([\mathrm{M}+\mathrm{H}]^{+}\right.$, $\mathrm{C}_{35} \mathrm{H}_{39} \mathrm{Cl}_{2} \mathrm{~N}_{8} \mathrm{O}_{4}, 705.2471$ ) was used. The procedure for accurate mass determination on the TSQ Quantum Ultra AM involved linearising the mass range using the calibration solution and then a refining of the accurate mass measurement using the reference lock masses. The lock masses calibration mixtures of reference compounds of known $\mathrm{m} / \mathrm{z}$ in acetonitrile were infused by syringe pump as a sheath liquid at a flow rate of $5 \mu \mathrm{L} / \mathrm{min}$. The Selected Ion Monitoring (SIM) scan was performed at a peak width of $0.2 \mathrm{Da}$ 
FWHM on Q3 in internal calibration mode using the AutoLock option at a scan rate of $10 \mathrm{amu} / \mathrm{s}$ and with a scan width of $0.8 \mathrm{amu}$. The AutoLock procedure automatically brackets the most abundant ion of interest with two lock masses from the reference list and mass measures the ion of interest. All the experimentally-determined accurate mass measurements resulted in only one proposed elemental composition for all tested compounds and showed satisfactory results in terms of accuracy (lower than 4.7 ppm). Moreover, for some compounds the calculation of the exact masses has been carried out using the most abundant halogen isotopes (i.e. ${ }^{35} \mathrm{Cl}$ and ${ }^{79} \mathrm{Br}$ ).

Accurate Mass and analytical HPLC data.

\begin{tabular}{|c|c|c|c|c|c|c|}
\hline Compd & $\begin{array}{c}\text { Elemental } \\
\text { composition } \\
\text { generated }[\mathrm{M}+\mathrm{H}]+\end{array}$ & $\begin{array}{c}\text { Theoretica } \\
1 \text { Mass } \\
{[\mathrm{M}+\mathrm{H}]+}\end{array}$ & $\begin{array}{c}\text { Accurate } \\
\text { Molecular weight } \\
{[\mathrm{M}+\mathrm{H}]+}\end{array}$ & $\begin{array}{l}\text { Delta } \\
(\mathrm{ppm})\end{array}$ & $\begin{array}{l}\text { Retention time } \\
\text { Method } 1^{a}\end{array}$ & $\begin{array}{l}\text { Retention time } \\
\text { Method } 2^{a}\end{array}$ \\
\hline 11 & C19H24FN4O3 & 375.1827 & 375.18338 & 1.82374 & 0.61 & 0.91 \\
\hline 16 & C18H22FN4O4 & 377.16196 & 377.16019 & -4.70222 & 0.59 & 0.69 \\
\hline 19 & $\mathrm{C} 25 \mathrm{H} 28 \mathrm{FN} 4 \mathrm{O} 4$ & 467.20891 & 467.20829 & -1.33446 & 0.69 & 1.3 \\
\hline 36 & C23H31FN5O5 & 476.23037 & 476.23018 & -0.4004 & 0.71 & 1.28 \\
\hline 27 & C18H22FN4O4 & 377.16196 & 377.16258 & 1.63111 & 0.61 & 0.93 \\
\hline 10 & C18H22FN4O3 & 361.16705 & 361.16811 & 2.94333 & 0.61 & 0.94 \\
\hline 33 & C18H23FN5O3 & 376.17794 & 376.17758 & -0.96463 & 0.59 & 0.91 \\
\hline 34 & C20H27FN5O3 & 404.20924 & 404.20789 & -3.3623 & 0.61 & 0.98 \\
\hline 38 & C20H25FN5O4 & 418.18851 & 418.18847 & -0.08738 & 0.57 & 0.85 \\
\hline 35 & C21H29FN5O3 & 418.22489 & 418.22428 & -1.4791 & 0.62 & 1.04 \\
\hline $27 \mathbf{b}$ & C18H22FN4O4 & 377.16196 & 377.16304 & 2.87066 & 0.61 & 0.92 \\
\hline 46 & C17H20FN4O4 & 363.14631 & 363.14641 & 0.28408 & 0.59 & 0.87 \\
\hline 47 & C19H24FN4O4 & 391.17761 & 391.17661 & -2.56944 & 0.61 & 0.94 \\
\hline 48 & $\mathrm{C} 19 \mathrm{H} 22 \mathrm{FN} 4 \mathrm{O} 5$ & 405.15687 & 405.15787 & 2.44975 & 0.83 & 1.27 \\
\hline 42 & C19H25FN5O5S & 454.1555 & 454.15451 & -2.17864 & 0.66 & 1.02 \\
\hline 28 & $\mathrm{C} 18 \mathrm{H} 22 \mathrm{FN} 4 \mathrm{O} 3 \mathrm{~S}$ & 393.13912 & 393.1387 & -1.05567 & 0.62 & 0.98 \\
\hline 29 & $\mathrm{C} 18 \mathrm{H} 22 \mathrm{FN} 4 \mathrm{O} 4 \mathrm{~S}$ & 409.13403 & 409.13428 & 0.59953 & 0.66 & 0.99 \\
\hline 49 & C22H28FN4O6 & 463.19874 & 463.19693 & -3.90725 & 1 & 1.74 \\
\hline 41 & C21H28FN6O4 & 447.21506 & 447.21529 & 0.5268 & 0.61 & 0.97 \\
\hline 37 & C20H25FN5O4 & 418.18851 & 418.18826 & -0.58929 & 0.61 & 0.92 \\
\hline 39 & $\mathrm{C} 25 \mathrm{H} 27 \mathrm{FN} 5 \mathrm{O} 4$ & 480.20416 & 480.2034 & -1.58223 & 0.69 & 1.18 \\
\hline 40 & C22H30FN6O4 & 461.23071 & 461.23105 & 0.73719 & 0.5 & 0.77 \\
\hline 43 & C24H27FN5O5S & 516.17115 & 516.17212 & 1.88552 & 0.78 & 1.39 \\
\hline 17 & C19H24FN4O4 & 391.17761 & 391.17788 & 0.69471 & 0.61 & 0.95 \\
\hline
\end{tabular}




$\begin{array}{ccccccc}\mathbf{1 8} & \text { C20H26FN4O4 } & 405.19326 & 405.19352 & 0.64632 & 0.62 & 1.02 \\ \mathbf{2 4} & \text { C18H20F3N4O3 } & 397.1482 & 397.14844 & 0.59287 & 0.78 & 1.31 \\ \mathbf{2 0} & \text { C19H25FN5O5S } & 454.1555 & 454.15433 & -2.57274 & 0.61 & 0.93 \\ \mathbf{2 1} & \text { C20H25FN5O4 } & 418.18851 & 418.18854 & 0.06973 & 0.59 & 0.93 \\ \mathbf{2 2} & \text { C18H21F2N4O3 } & 379.15762 & 379.15775 & 0.32053 & 0.61 & 0.96 \\ \mathbf{2 6} & \text { C26H30FN4O4 } & 481.22456 & 481.22476 & 0.41854 & 0.71 & 1.32 \\ \mathbf{2 5} & \text { C19H24FN4O4 } & 391.17761 & 391.17736 & -0.64957 & 0.59 & 0.87 \\ \mathbf{2 3} & \text { C18H21F2N4O3 } & 379.15762 & 379.15778 & 0.40102 & 0.59 & 0.91 \\ \mathbf{1 3} & \text { C19H24FN4O3 } & 375.1827 & 375.18355 & 2.28862 & 0.62 & 1.01 \\ \mathbf{1 0 a} & \text { C18H22FN4O3 } & 361.16705 & 361.16635 & -1.92238 & 0.61 & 0.93 \\ \mathbf{4 4} & \text { C20H28FN6O5S } & 483.18204 & 483.18173 & -0.64728 & 0.68 & 1.13 \\ \mathbf{1 4} & \text { C19H24FN4O3 } & 375.1827 & 375.18201 & -1.83605 & 0.62 & 1.01 \\ \mathbf{1 5} & \text { C19H24FN4O3 } & 375.1827 & 375.18213 & -1.5193 & 0.61 & 0.97 \\ \mathbf{1 2} & \text { C19H24FN4O3 } & 375.1827 & 375.18308 & 1.03418 & 0.62 & 0.99 \\ \mathbf{3 2} & \text { C19H23FN5O4 } & 404.17286 & 404.17237 & -1.20311 & 0.74 & 1.17 \\ \mathbf{5 9} & \text { C19H24FN4O4 } & 391.17761 & 391.17744 & -0.44817 & 0.64 & 1.05 \\ \mathbf{5 0} & \text { C18H23N4O4 } & 359.17138 & 359.17157 & 0.52282 & 0.57 & 0.86 \\ \mathbf{5 3} & \text { C18H22FN4O4 } & 377.16196 & 377.16336 & 3.71282 & 0.59 & 0.88 \\ \mathbf{5 8} & \text { C18H21F2N4O4 } & 395.15254 & 395.15317 & 1.59243 & 0.62 & 0.98 \\ \mathbf{5 5} & \text { C18H22ClN4O4 } & 393.13217 & 393.13241 & -0.60566 & 0.64 & 1.03 \\ \mathbf{5 7} & \text { C19H25N4O5 } & 389.18195 & 389.18161 & -0.86162 & 0.59 & 0.9 \\ \mathbf{5 6} & \text { C18H22BrN4O4 } & 437.08295 & 437.08189 & 2.41069 & 0.64 & 1.06 \\ \mathbf{5 4} & \text { C18H22FN4O4 } & 377.16196 & 377.16116 & -2.11296 & 0.59 & 0.91 \\ \mathbf{6 0} & \text { C18H21ClFN4O4 } & 411.12299 & 411.12301 & 0.06579 & 0.66 & 1.08 \\ \mathbf{5 1} & \text { C19H25N4O4 } & 373.18703 & 373.18771 & 1.82617 & 0.61 & 0.95 \\ \mathbf{5 2} & \text { C19H26N4O4 } & 373.18703 & 373.18665 & -1.03597 & 0.54 & 0.79\end{array}$

${ }^{a}$ Method 1: Stationary phase: Waters Sunfire $\mathrm{C}_{18}$ column, $5 \mu \mathrm{m}, 30$ x $2.1 \mathrm{~mm}$. Mobile phase consisting of a solvent $\mathrm{A}$ (water $+0.1 \%$ formic acid) and solvent B (acetonitrile $+0.1 \%$ formic acid) was delivered at a flow rate of $1000 \mu \mathrm{L} / \mathrm{min}$. The LC gradient started from 95/5\% (A/B) and changed to 5/95\% (A/B) at 1.0 min, remaining constant to this ratio until $1.5 \mathrm{~min}$. The gradient increased to $95 / 5 \%(\mathrm{~A} / \mathrm{B})$ at $1.6 \mathrm{~min}$, remaining constant to this ratio until 2.0 min that was the total analysis time.

${ }^{b}$ Method 2: Stationary phase: Acquity UPLC ${ }^{\mathrm{TM}}$ BEH $\mathrm{C}_{18} 1.7 \mu \mathrm{m}, 2.1$ x $50 \mathrm{~mm}$ column. Mobile phase comprised a linear gradient of binary mixtures of $\mathrm{H}_{2} \mathrm{O}$ containing $0.1 \%$ Formic Acid (solvent A) and MeCN containing 0.1\% Formic Acid (solvent B). Flow rate $0.5 \mathrm{ml} / \mathrm{min}$. Gradient: 10\% solvent B (0.1 
$\min$ ) to $100 \%$ solvent B over $2.5 \mathrm{~min}$, then 0.2 min isocratic; from $100 \%$ of solvent B to $10 \%$ over 0.1 $\min$.

References:

1) Zhang, Z.; Van Aerschot, A.; Hendrix, C.; Busson, R.; David, F.; Sandra, P.; Herdewijn, P. Tetrahedron 2000, 56, 2513.

2) Demange, L.; Menez, A.; Dugave, C. Practical Synthesis of Boc and Fmoc Protected 4-Fluoro and 4-Difluoroprolines from trans-4-Hydroxyproline. Tetrahedron Letters 1998, 39, 1169-1172. 